\title{
On steady distributions of kinetic models of conservative economies
}

\author{
Daniel Matthes * Giuseppe Toscani ${ }^{\dagger}$
}

October 22, 2007

\begin{abstract}
We analyze the large-time behavior of various kinetic models for the redistribution of wealth in simple market economies introduced in the pertinent literature in recent years. As specific examples, we study models with fixed saving propensity introduced by A. Chakraborty and B.K. Chakrabarthi [11], as well as models involving both exchange between agents and speculative trading as considered by S. Cordier, L. Pareschi and one of the authors [14]. We derive a sufficient criterion under which a unique non-trivial stationary state exists, and provide criteria under which these steady states do or do not possess a Pareto tail. In particular, we prove the absence of Pareto tails in pointwise conservative models, like the one in [11], while models with speculative trades introduced in [14] develop fat tails if the market is "risky enough". The results are derived by a Fourier-based technique first developed for the Maxwell-Boltzmann equation [26, 1, 39], and from a recursive relation which allows to calculate arbitrary moments of the stationary state.
\end{abstract}

Keywords. Econophysics, Boltzmann equation, wealth and income distributions, Pareto distribution.

\section{Introduction}

In recent years, various kinetic models of Boltzmann type have been introduced to study the evolution of the distribution of wealth in a simple market economy [30, 20, 11, 10, 29, 47, 14]. In most of these models, the market is represented as an ideal gas, agents correspond to molecules, and each trading event between two agents is considered to be an elastic - or rather money conserving - collision of two molecules. The founding idea is that a trading market composed of a sufficiently large number of agents can essentially be described using the same laws of statistical mechanics which govern the behavior of a physical system composed of many interacting particles.

In these models, the knowledge of the large-wealth behavior of the steady state density is of primary importance, since it determines a posteriori if the model fits data of real economies.

\footnotetext{
*Fachbereich Mathematik und Informatik Johannes Gutenberg-Universität, Staudingerweg 9, 55099 Mainz, Germany. matthes@mathematik.uni-mainz.de

$\dagger$ Department of Mathematics, University of Pavia, via Ferrata 1, 27100 Pavia, Italy.

giuseppe.toscani@unipv.it
} 
More than a hundred years ago, the Italian economist Vilfredo Pareto [40] first quantified the large-wealth behavior of the income distribution in a society and found it to follow a powerlaw distribution. More precisely, if $f(w)$ denotes the density of agents with wealth $w$, and $w$ is sufficiently large, then

$$
\int_{w}^{\infty} f\left(w_{*}\right) d w_{*} \sim w^{-\mu}
$$

Pareto mistakenly believed that power laws apply to the whole distribution with a universal exponent $\mu$ approximatively equal to 1.5. Later, Mandelbrot proposed a weak Pareto law that applies only to high incomes [36]. The exponent $\mu$, usually called the Pareto index, was initially found to have a value between 1 and 3. Numerous investigations with real data during the last ten years revealed that the tails of the income distribution indeed behave as indicated above, and the value of the Pareto index varies between 1 and 2.5 (USA $\sim 1.6$, Japan $\sim 1.8-2.2[20,34,21]$ ). Typically, the weak Pareto law applies to the richest $10 \%$ of the population - which possesses about $40 \%$ of the total wealth of that country. The mid- and low-income population, in fact the majority (90\% or more), follow a different distribution which is debated to be either Gibbs [33, 30, 20] or log-normal [18].

In any kinetic description, the dynamics is defined through the microscopic interactions. In the econophysical context, the model is specified by the rules according to which agents perform their trades. A seemingly important and very sensible ingredient is the saving factor. This means that each of the interacting individuals keeps a certain fraction of his current wealth, and only puts the remaining part into the trade - at the risk of losing it. The first extensive studies of this class of models are probably due to Chakraborti and Chakrabarti [11], and to Chatterjee, Chakrabarti and Manna [12]. Indeed, some of their numerical experiments reproduce a wealth distribution similar to that of a real economy. Mainly two variants to introduce saving propensities have been studied: In the first, all agents have the same fixed saving factor [11], while in the second, the saving propensity has a quenched random distribution [12]. Numerous simulations have been carried out for both types of model, but few mathematical rigorous results are available up to now: The fixed-rate model has been understood to a certain extent (see e.g, [15, 41]); its stationary state resembles a gamma distribution [41]. On the other hand, there exists no complete analysis for the model with randomly distributed saving factors (see however, [44, 38, 13, 37]).

A somewhat different approach to trades was made by Cordier, Pareschi, and the second author in [14]. There, a very simple model of an open market economy involving both assets exchanges between individuals and speculative trading was introduced. The model is such that the average wealth is not conserved due to a random dynamics in the binary trade interactions. The latter describes the spontaneous growth or decrease of wealth related to investments in the stock market, housing, and other factors. In the absence of conservation of the total wealth, the best way to extract information on the large-time behavior is to scale the solution in a way which keeps the total wealth constant. Nevertheless, analytical studies of the resulting kinetic model remained difficult (although a thorough analysis of the continuous trading limit has been performed). For a determination of the shape of the limit distribution, the authors made use of suitable numerical methods.

Concerning the use of numerical methods in the study of steady states to kinetic equations (and in particular the tails of these solutions), we recall that one is faced with delicate problems. In fact, 
the numerical reconstruction of tails in self-similar profiles of non-linear evolution equations has a long history, especially in the field of diffusion processes. The main numerical difficulty originates from the so-called retention property, which means that the support of the solution is growing in time. Indeed, because of its spreading dynamics, diffusion equations will ask for repetitive regridding. For some recent developments in this area we refer to [28], where a new tool has been proposed, which allows to achieve precise numerical results in the case of fast diffusion equations. An analogous problem exists in the kinetic context, and has been recently apparent in the studies of the long-time behavior of a dissipative Maxwell gas [23, 24]. Despite the fact that the selfsimilar profiles are know to exhibit overpopulated tails, a numerical reconstruction of the latter is difficult to obtain. The available methods, principally Montecarlo ones, are oscillating exactly in the interesting large velocities part of the density profile [45, 27].

In conclusion of the preceding remarks, one should treat the output of numerical experiments with care, when it comes to determine the structure of tails. Rigorous analytical information is of high importance in this discussion. The intention of the present paper is to bridge the gap between the numerical observations and their analytical understanding, at least for some of the available models. First of all, we investigate under which assumptions on the model one may rightfully speak of a stationary wealth distribution. More precisely, we prove the existence and uniqueness of a steady state, and we obtain results about the convergence of the dynamics towards it in the long-time limit. Subsequently, we investigate the regularity of the steady state. We confirm the expectation that a sensible wealth distribution curve is smooth. Finally - and most importantly we provide a method to calculate the Pareto index directly from the model parameters.

In order to extract information on the large-time behavior of the economic system, we adopt ideas from [39]. The two main ingredients are the use of Fourier metrics and estimates on the evolution of moments. In fact, this strategy is well-known in dissipative kinetic theory, where the large-time behavior of the solution to the dissipative Boltzmann equation for Maxwell molecules [4] has been investigated by exactly the same means [23, 24, 1]. We would like to stress, however, that our investigations here go beyond an application of the mentioned results. In fact, the models of interest here do not fit into the "classical picture" of kinetic equations. As we do not allow debts, the wealth of our agents is always positive; this corresponds to a kinetic model which forbids negative velocities. In particular, we cannot assume that the distribution function is isotropic. Moreover, the role played by the second moment, i.e. the energy, in the classical picture is now played the first moment, which is the mean wealth of the society.

For the sake of completeness, we mention that alternative strategies have been used before in order to obtain analytical information about the tails of the steady wealth distribution. For instance, in [14], the kinetic equation was replaced by a (simpler) Fokker-Planck-type equation. This was justified by proving that the latter constitutes the grazing collisions limit of the first. The equilibrium state of the Fokker-Planck equation can be computed explicitly and is of Pareto type, i.e. it exhibits the power-law tail for the richest individuals. The same Fokker-Planck equation was obtained in [7] as the mean field limit of a stochastic dynamical equation, as well as in $[48,35]$ in the context of a generalized Lotka-Volterra dynamic. The mathematical methods used in [14] are close to those used in the context of kinetic theory for granular flows. Analogous methods have been used for a kinetic model of a simple market economy with a constant growth mechanism by Slanina [47], and generalized to a one-dimensional kinetic model with mixing interaction in [39]. Finally, the hydrodynamic limit of a market model with time-dependent saving has been derived 
and studied numerically in [22].

Below, we provide more specific description of the setup and summary of our results.

In section 2, we define the class of models to study. The time evolution of the wealth distribution $f(v, t)$ is based on the assumption of collision-like trade events. In a suitable scaling, the effect of collisions is quantitatively described by a Boltzmann-like equation [9],

$$
\frac{\partial f}{\partial t}=Q(f, f)
$$

Here $f=f(v, t)$ is the distribution of wealth $v \in \mathbb{R}_{+}$at time $t \geq 0$, and $Q$ is a bilinear operator which describes the change of $f$ due to trades among agents. The binary trade is determined by the linear exchange rules

$$
v^{*}=p_{1} v+q_{1} w ; \quad w^{*}=p_{2} v+q_{2} w .
$$

Here $(v, w)$ denote the (positive) money of two arbitrary individuals before the trade, and $\left(v^{*}, w^{*}\right)$ the money after the trade. The interaction coefficients $p_{i}, q_{i}, i=1,2$ can be either given constant or random quantities, with the obvious constraint to be nonnegative. In the present paper - except for section 4.2 - the random distribution (or collision kernel, in the kinetic language) is assumed to be independent of the wealth variables $v$ and $w$, and independent of time. Moreover, we restrict ourselves to conservative models, characterized by the further property

$$
\left\langle p_{1}+p_{2}\right\rangle=1, \quad\left\langle q_{1}+q_{2}\right\rangle=1,
$$

where $\langle\cdot\rangle$ denotes the expectation value. Such interactions define a dynamic for the distribution functions $f(v, t)$, i.e. for appropriate initial conditions, there exists a unique solution. This solution is continuous in a suitable Fourier metric for probability measures, see Theorem 2.3.

In section 3, we study the large-time behavior of solutions to (2), in particular their convergence towards a steady state, and the qualitative properties of the latter. The main result of this paper is given in Theorem 3.3: generically, the distribution functions $f(v, t)$ do converge towards a steady state $f_{\infty}(v)$ as $t \rightarrow \infty$, and this steady state is unique. Moreover, we prove that the Pareto index $\mu$ of $f_{\infty}$ in (1) is given by the non-trivial root $\bar{s}>1$ of the quantity

$$
\mathfrak{S}(s):=\frac{1}{2}\left\langle\sum_{i=1}^{2}\left(p_{i}^{s}+q_{i}^{s}\right)\right\rangle-1 .
$$

This quantity is directly accessible once the interaction coefficients in (3) are made explicit. If no root $\bar{s}>1$ exists, then the limiting distribution either possesses a slim tail, which decays superalgebraically as $v \rightarrow \infty$, or is trivial, i.e. all agents have wealth zero. Finally, Theorem 3.5 provides $C^{\infty}$-smoothness of the steady state under very general assumptions. In fact, the steady state belongs to a Gevrey class.

In addition to Fourier metrics, the other key tool in the proof of Theorem 3.3 is an investigation of the evolution of moments. The results are summarized in Theorem 3.2: if $\mathfrak{S}(s)$ is negative, then the sth moment of $f(v, t)$ remains uniformly bounded in time; if $\mathfrak{S}(s)$ is positive, then the $s$ th moment diverges exponentially fast as $t \rightarrow \infty$. 
In section 4 , the behavior of the function $\mathfrak{S}$ in various special cases is analyzed, and additional information about the shape of the tails is derived. For instance, in example 2, we choose the collision kernel in a way that allows to control its degree of randomness by a parameter $\mu$. The typical behavior we find (see Figure 1) is that for small randomness, the tails of the steady state decay super-algebraically; in fact, they decay exponentially by Theorem 4.4. On the other hand, for large randomness, the steady state is trivial. Pareto tails form exactly in the intermediate regime. Likewise, a small saving propensity $\lambda$ results in slim tails, while for large values $\lambda \approx 1$, the stationary state collapses to into the trivial solution. We conclude that a good adjustment of both randomness and saving play a pivotal role in the definition of suitable models.

Another observation concerns pointwise conservative trades, where the total of money is conserved not only in the mean, but in each interaction. Then either the tail of $f_{\infty}$ is slim, or is possibly of Pareto type but unstable in the sense that it is reached at a slow algebraic time scale, but not exponentially fast; see Theorem 4.1 for details. Hence, in order to obtain a natural and robust wealth structure in the long-time limit, the respective model must allow the total money to increase during an individual trade with a certain probability; likewise, it must allow the total to decrease. This behavior is most easily interpreted as a combination of trades with risky investments, as discussed above in the context of the model of Cordier et al.

Finally, we mention how our results are useful to understand the outcome of numerical experiments. By Theorem 3.3, a variety of models allows only for trivial steady distribution of wealth, in which all agents are truly pauper. However, the convergence of the money distribution $f(v, t)$ towards this pathological limit $f_{\infty}(v)$ goes via the blow-up of all moments above the first, i.e. while an increasing majority of agents accumulates in the pauper region, fewer and fewer individuals become extremely rich, because the total wealth of the society is preserved. On the other hand, in the long-time limit, the total wealth is zero (the convergence is really weak in this case). In a simple numerical experiment, in which finite-size effects play a role, one could mistakenly interpret the blow-up of all moments above the first as convergence to a steady state with Pareto-index $\mu \approx 1$. This gives a possible explanation of why so many numerical studies report a critical index of approximately one, corresponding to a state with infinite mean wealth.

In summary, our results improve the understanding of the trade-mechanisms which are responsible for the creation of Pareto tails. In particular, we prove that the large-wealth behavior of the steady distribution heavily depends on the degree of randomness in the microscopic trade, and on the propensity of agents to save.

\section{The kinetic equation}

\subsection{Collision rules in conservative economies}

A kinetic model of a simple market economy describes the redistribution of wealth by means of microscopic binary interactions among agents or individuals which perform exchange of money. These interactions are interpreted as trades. One generally assumes that the outcome of each trade is non-negative for both agents, imposing that no debts are allowed. This rule emphasizes the difference between economic interactions and the classical interactions between molecules, where no positivity restriction applies. 
On the microscopic level, we consider binary interactions described by the rules (3), i.e.,

$$
v^{*}=p_{1} v+q_{1} w ; \quad w^{*}=p_{2} v+q_{2} w,
$$

where $(v, w)$ and $\left(v^{*}, w^{*}\right)$ denote the money of two arbitrary individuals before and after the trade, respectively. The interaction coefficients $p_{i}, q_{i}$ for $i=1,2$ can be either given constant or random quantities. Here random means with respect to an a priori given probability distribution for the $p_{i}$ and $q_{i}$, which does not depend on time $t$, or on $v$ and $w$. As debts are not allowed, the interaction coefficients are constrained to be nonnegative. The total amount of money involved in the trade changes from $v+w$ into

$$
v^{*}+w^{*}=\left(p_{1}+p_{2}\right) v+\left(q_{1}+q_{2}\right) w
$$

so that, unless a.s. both

$$
p_{1}+p_{2}=1, \quad q_{1}+q_{2}=1,
$$

money is generally not conserved. Condition (7) can be relaxed in presence of random coefficients: we require that the total money is conserved in the mean, that is the random coefficients $p_{i}, q_{i}$, $i=1,2$ satisfy

$$
\left\langle p_{1}+p_{2}\right\rangle=1, \quad\left\langle q_{1}+q_{2}\right\rangle=1 .
$$

Both conditions (7) and (8) define conservative trades, and we refer to an interaction with (7) as pointwise conservative. These interactions are such that the mean wealth

$$
M=\int v f(v) d v
$$

is conserved in time.

Remark 2.1 We shall frequently use the abbreviation

$$
\left\langle\Psi\left(p_{i}, q_{i}\right)\right\rangle_{+}:=\frac{1}{2}\left\langle\Psi\left(p_{1}, q_{1}\right)+\Psi\left(p_{2}, q_{2}\right)\right\rangle
$$

for expressions $\Psi$ involving $p_{i}$ and $q_{i}$. Note that (8) implies the weaker condition

$$
\left\langle p_{i}+q_{i}\right\rangle_{+}=1
$$

which will be sufficient for most of our considerations.

Both types of conservative interaction allow for a similar (and almost complete) study. Various models in the literature define conservative trades. In their pioneering paper A. Chakraborty and B.K. Chakrabarthi [11] imposed that the agents taking part in a trade change money according to the rule

$$
v^{*}=v+\Delta(v, w) ; \quad w^{*}=w-\Delta(v, w),
$$

where $\Delta(v, w)$ represents the amount of money to be exchanged,

$$
\Delta(v, w)=(1-\lambda)[(\varepsilon-1) v+\varepsilon w]
$$


with a constant saving propensity $\lambda \in[0,1]$, and a random fraction $\varepsilon$ with given distribution on $[0,1]$. The introduction of $\lambda$ in the definition of $\Delta(v, w)$ takes into account the basic fact that rational agents always retain some money when trading; the saving rate $\lambda$ (or sometimes $s$ ) denotes the ratio of the withheld to all the money possessed. This trade corresponds to

$$
\begin{aligned}
& p_{1}=1-(1-\lambda)(1-\varepsilon), \quad q_{1}=(1-\lambda) \varepsilon \\
& p_{2}=(1-\lambda)(1-\varepsilon), \quad q_{2}=1-(1-\lambda) \varepsilon
\end{aligned}
$$

and is pointwise conservative.

We remark that the model has been further developed [12]: instead of prescribing a "global" saving rate for all agents, an individual saving propensity $\lambda_{i} \in[0,1]$ is randomly assigned to each individual agent. This rate is then held fix during the time evolution. The rules (13) change accordingly into

$$
\begin{aligned}
p_{1}=1-\left(1-\lambda_{A}\right)(1-\varepsilon), & q_{1}=\left(1-\lambda_{B}\right) \varepsilon \\
p_{2}=\left(1-\lambda_{A}\right)(1-\varepsilon), & q_{2}=1-\left(1-\lambda_{B}\right) \varepsilon
\end{aligned}
$$

where the quantities $\lambda_{A}$ and $\lambda_{B}$ denote the saving rates of the two interacting individuals $A$ and $B$, possessing the wealth $v$ and $w$, respectively. Despite its pointwise conservative character, this model does not fit into the current framework. Note that the interaction coefficients are not distributed according to an a priori law; rather, finding the (time- and wealth-depending) law for $\lambda$ becomes part of the problem here. Some remarks are given in section 4.2. However, a thorough analysis of this model will be subject of a separate paper [37].

Another kind of trade was considered in [14]. Its rules read

$$
v^{*}=\lambda v+(1-\lambda) w+\eta v ; \quad w^{*}=(1-\lambda) v+\lambda w+\tilde{\eta} w .
$$

The saving rate $\lambda$ is a global constant, as in the rules (13), while $\eta$ and $\tilde{\eta}$ are independent random variables with the same distribution and zero mean. This random term introduces the effects of an open economy describing the market returns. Provided that $\eta, \tilde{\eta} \geq-\lambda$ almost surely, the random coefficients $p_{i}, q_{i}, i=1,2$ in (15) are nonnegative, since

$$
\begin{aligned}
& p_{1}=\lambda+\eta, \quad q_{1}=1-\lambda \\
& p_{2}=1-\lambda, \quad q_{2}=\lambda+\tilde{\eta} .
\end{aligned}
$$

However, this trade is conservative only in the mean, since $\left\langle p_{1}+p_{2}\right\rangle=1$, but $p_{1}+p_{2}=1+\eta \neq 1$ with probability one.

For the sake of completeness, we mention some non-conservative models. F. Slanina recently introduced an increasing wealth model [47] where

$$
p_{1}=\lambda, \quad q_{1}=1-\lambda+\varepsilon ; \quad p_{2}=1-\lambda+\varepsilon, \quad q_{2}=\lambda .
$$

In the rules (17), the growth parameter $\varepsilon$ is a fixed positive constant, hence the total money has increased after the trade,

$$
v^{*}+w^{*}=(1+\varepsilon)(v+w) .
$$


These rules introduce (artificially) the effects of a strong economy into the game, making the total wealth grow with time. The same effect can be obtained in a more natural way: allow the random variables in (15) to attain arbitrary real values, but discard all trades for which the post-trade money are negative (see the discussion in [14]). The same idea was used in the original transfer model proposed by A. Drăgulesku and V. Yakovenko [20], where the amount of money exchanged is

$$
\Delta(v, w)=\frac{\varepsilon}{2}(v+w)
$$

with a random number $0 \leq \varepsilon \leq 1$. If the "loser" cannot afford the money to be exchanged, the trade does not take place.

Non-conservative kinetic models for trades of the type considered by Slanina [47] have been recently studied in [39] by means of mathematical methods first applied to the Boltzmann equation for Maxwell molecules. In the forthcoming section, we will show how these techniques generalize to treat the conservative models introduced so far.

\subsection{Weak form of the Boltzmann equation}

Let $f(v, t)$ denote the distribution of agents with wealth $v \in \mathbb{R}_{+}$at time $t \geq 0$. A Boltzmann-like equation for the time evolution of $f(v, t)$ can be easily derived by standard methods of kinetic theory, considering that the temporal change of $f(v, t)$ depends on a balance between the gain and loss of wealth among agents due to trades. A useful way of writing this equation is to use the so-called weak form: for all smooth functions $\Phi(v)$, consider

$$
\frac{d}{d t} \int_{\mathbb{R}_{+}} f(v) \Phi(v) d v=\int_{\mathbb{R}_{+}} Q(f, f)(v) \Phi(v) d v,
$$

where the collision operator $Q(f, f)$ is defined by

$$
\int_{\mathbb{R}_{+}} Q(f, f)(v) \Phi(v) d v=\frac{1}{2}\left\langle\int_{\mathbb{R}_{+}} \int_{\mathbb{R}_{+}}\left(\Phi\left(v^{*}\right)+\Phi\left(w^{*}\right)-\Phi(v)-\Phi(w)\right) f(v) f(w) d v d w\right\rangle .
$$

The kinetic equation (20) is the analogue of the Boltzmann equation for Maxwell molecules [3,9], where the collision frequency is assumed to be constant. It is well-known in kinetic theory that this simplification allows for a better understanding of the qualitative behavior of the solutions. Among other properties, it can be shown that higher order moments can be evaluated recursively, since the integrals $\int v^{n} f(v, t) d v$ obey a closed hierarchy of equations.

Without loss of generality, we can fix the initial density to satisfy

$$
\int_{\mathbb{R}_{+}} f_{0}(v) d v=1 ; \quad \int_{\mathbb{R}_{+}} v f_{0}(v) d v=M .
$$

Choosing $\Phi(v)=1$, (respectively $\Phi(v)=v$ ) into (20) shows that conditions (22) are propagated in time, so that, for all $t>0$

$$
\int_{\mathbb{R}_{+}} f(v, t) d v=1 ; \quad \int_{\mathbb{R}_{+}} v f(v, t) d v=M
$$


The initial value problem can be easily studied in the weak form (20). Denote by $\mathscr{M}_{0}$ the space of all probability measures in $\mathbb{R}_{+}$, and for $\alpha \geq 0$ by

$$
\mathscr{M}_{\alpha}=\left\{\mu \in \mathscr{M}_{0}: \int_{\mathbb{R}_{+}}|v|^{\alpha} \mu(d v)<+\infty\right\}
$$

the space of all Borel probability measures of finite moment of order $\alpha$, equipped with the topology of the weak convergence of measures.

A weak solution to the initial value problem for equation (20), with initial probability density $f_{0}(v) \in \mathscr{M}_{\alpha}, \alpha>0$ is a probability density $f \in C^{1}\left(\mathbb{R}_{+}, \mathscr{M}_{\alpha}\right)$ which satisfies (20) for $t>0$ and all smooth functions $\Phi$, and the initial condition is realized as

$$
\lim _{t \rightarrow 0} \int_{\mathbb{R}_{+}} \Phi(v) f(v, t) d v=\int_{\mathbb{R}_{+}} \Phi(v) f_{0}(v) d v
$$

for all $\Phi$.

\subsection{The Boltzmann equation in Fourier representation}

From the kinetic theory of rarefied gases it is well-known that Boltzmann-like equations are often conveniently studied in Fourier space, see e.g. [3]. This is particularly true for the model under consideration, since its collision kernel (the probability measure) is independent of $v$. In fact, the weak formulation (20) is equivalent to the Fourier transformed equation:

$$
\frac{\partial \widehat{f}(\xi, t)}{\partial t}=\widehat{Q}(\widehat{f}, \widehat{f})(\xi, t)
$$

where $\widehat{f}(\xi, t)$ is the Fourier transform of $f(x, t)$,

$$
\widehat{f}(\xi, t)=\int_{\mathbb{R}_{+}} e^{-i \xi v} f(v, t) d v .
$$

According to the collision rule (5), the transformed kernel reads

$$
\widehat{Q}(\widehat{f}, \widehat{f})(\xi)=\frac{1}{2}\left\langle\widehat{f}\left(p_{1} \xi\right) \widehat{f}\left(q_{1} \xi\right)+\widehat{f}\left(p_{2} \xi\right) \widehat{f}\left(q_{2} \xi\right)\right\rangle-\widehat{f}(\xi) \widehat{f}(0) .
$$

The initial conditions (22) turn into

$$
\widehat{f}_{0}(0)=1 \quad \text { and } \quad \widehat{f}_{0}^{\prime}(0)=i M .
$$

Hence equation (26) can be rewritten as

$$
\frac{\partial \widehat{f}(\xi, t)}{\partial t}+\widehat{f}(\xi, t)=\left\langle\widehat{f}\left(p_{i} \xi\right) \widehat{f}\left(q_{i} \xi\right)\right\rangle_{+}
$$

Equation (28) is a generalization of equation (43) in [39], as we allow random variables for the mixing parameters $p_{i}$ and $q_{i}$. Further, (28) is connected to equation (4.8) considered by Bobylev and Cercignani in [5]. The difference is that in our situation $v$ is restricted to the positive real 
axis, which makes it impossible to apply most of the techniques from [5]. In particular, we cannot rescale our model in such a way that both the first and the second moment are preserved.

To prove existence of solutions to equation (28) is rather classical; e.g., the same methods available for the elastic Kac model [31] apply, expressing the solution as a Wild sum [3]. In order to show uniqueness of the solution, we use the idea from [26]: it is proven that the collision operator $Q$ is Lipschitz continuous in a suitable metric.

Introduce for arbitrary $s>0$ the Fourier metric on $\mathscr{M}_{\alpha}$ by

$$
d_{s}(f, g)=\sup _{\xi \in \mathbb{R}} \frac{|\widehat{f}(\xi)-\widehat{g}(\xi)|}{|\xi|^{s}} .
$$

Write $s=m+\beta$, where $m$ is an integer and $0<\beta \leq 1$. Two functions $f$ and $g$ have a finite distance, $d_{s}(f, g)<\infty$, if and only if their moments up to order $m$ agree.

The metric (29) has been introduced in [26] to investigate the trend to equilibrium of the solutions to the Boltzmann equation for Maxwell molecules. There, the case $s=2+\beta$ with $\beta>0$ was considered. Further applications of $d_{s}$ to various kinetic models can be found in $[1,39,43]$.

In order to show Lipschitz-continuity, let $f_{1}$ and $f_{2}$ be two solutions of the kinetic equation (28), corresponding to initial values $f_{1,0}$ and $f_{2,0}$ satisfying conditions (22), and denote by $\widehat{f}_{1}, \widehat{f}_{2}$ their Fourier transforms. Let $s \geq 1$ be such that $d_{s}\left(f_{1,0}, f_{2,0}\right)$ is finite. Then

$$
\frac{\partial}{\partial t} \frac{\left(\widehat{f}_{1}(\xi)-\widehat{f}_{2}(\xi)\right)}{|\xi|^{s}}+\frac{\widehat{f}_{1}(\xi)-\widehat{f}_{2}(\xi)}{|\xi|^{s}}=\frac{\left\langle\widehat{f}_{1}\left(p_{i} \xi\right) \widehat{f}_{1}\left(q_{i} \xi\right)-\widehat{f}_{2}\left(p_{i} \xi\right) \widehat{f}_{2}\left(q_{i} \xi\right)\right\rangle_{+}}{|\xi|^{s}} .
$$

Now, since $\left|\widehat{f}_{1}(\xi, t)\right| \leq 1$ and $\left|\widehat{f}_{2}(\xi, t)\right| \leq 1$ for all $\xi \in \mathbb{R}$, we obtain

$$
\begin{aligned}
& \left|\frac{\left\langle\widehat{f}_{1}\left(p_{i} \xi\right) \widehat{f}_{1}\left(q_{i} \xi\right)-\widehat{f}_{2}\left(p_{i} \xi\right) \widehat{f}_{2}\left(q_{i} \xi\right)\right\rangle_{+}}{|\xi|^{s}}\right| \\
& \quad \leq\left\langle\left|\widehat{f}_{1}\left(p_{i} \xi\right)\right|\left|\frac{\widehat{f}_{1}\left(q_{i} \xi\right)-\widehat{f}_{2}\left(q_{i} \xi\right)}{\left|q_{i} \xi\right|^{s}}\right| q_{i}^{s}\right\rangle_{+}+\left\langle\left|\widehat{f}_{2}\left(q_{i} \xi\right)\right|\left|\frac{\widehat{f}_{1}\left(p_{i} \xi\right)-\widehat{f}_{2}\left(p_{i} \xi\right)}{\left|p_{i} \xi\right|^{s}}\right| p_{i}^{s}\right\rangle_{+} \\
& \quad \leq \sup \left|\frac{\widehat{f}_{1}(\xi)-\widehat{f}_{2}(\xi)}{|\xi|^{s}}\right|\left\langle p_{i}^{s}+q_{i}^{s}\right\rangle_{+} .
\end{aligned}
$$

In terms of the auxiliary quantity

$$
h(t, \xi)=\frac{\widehat{f}_{1}(\xi)-\widehat{f}_{2}(\xi)}{|\xi|^{s}}
$$

the preceding computation shows that

$$
\left|\frac{\partial h}{\partial t}+h\right| \leq\left\langle p_{i}^{s}+q_{i}^{s}\right\rangle_{+}\|h\|_{\infty}
$$

Gronwall's lemma yields at once that

$$
\|h(t)\|_{\infty} \leq \exp \left\{\left(\left\langle p_{i}^{s}+q_{i}^{s}\right\rangle_{+}-1\right) t\right\}\left\|h_{0}\right\|_{\infty}
$$


Below and the following chapters, the quantity in round brackets,

$$
\mathfrak{S}(s)=\frac{1}{2} \sum_{i=1}^{2}\left\langle p_{i}^{s}+q_{i}^{s}\right\rangle-1,
$$

plays a major role. Since $\|h(t)\|_{\infty}=d_{s}\left(f_{1}(t), f_{2}(t)\right)$, we obtain from (31)

Theorem 2.2 Let $f_{1}(t)$ and $f_{2}(t)$ be two solutions of the Boltzmann equation (20), corresponding to initial values $f_{1,0}$ and $f_{2,0}$ satisfying conditions (22). Let $s \geq 1$ be such that $d_{s}\left(f_{1,0}, f_{2,0}\right)$ is finite. Then, for all times $t \geq 0$,

$$
d_{s}\left(f_{1}(t), f_{2}(t)\right) \leq \exp \{\mathfrak{S}(s) \cdot t\} d_{s}\left(f_{1,0}, f_{2,0}\right) .
$$

In particular, if $\mathfrak{S}(s)$ is negative, then the $d_{s}$-distance of $f_{1}$ and $f_{2}$ decays exponentially in time.

Putting $f_{1,0}=f_{2,0}=f_{0}$ in (33), and using $s=1$ yields

Corollary 2.3 If $f_{0}$ is a nonnegative density satisfying conditions (22), then there exists a unique weak solution $f(t)$ of the Boltzmann equation with $f(0)=f_{0}$.

\section{Existence, uniqueness and smoothness of the steady state}

In order to discuss the shape of the tail in the stationary distribution, we first need to establish the existence of the latter. We prove - under suitable hypotheses - convergence of the distribution function $f(v, t)$ to a long-time limit $f_{\infty}(v)$, and show that this constitutes the unique stationary state. We point out that the existence of the long-time limit cannot be directly concluded from the contractivity of the kinetic equation in some $d_{s}$-norm. Unfortunately, one needs additional information on the boundedness of higher moments; for further discussion on the lack of completeness w.r.t. Fourier distance, see [8]. Therefore, the temporal evolution of moments of $f(v, t)$ is investigated first. The second approach follows the idea of Bobylev and Cercignani [5] to approximate the steady state by using the stationary equation as an iteration scheme.

\subsection{Evolution of moments}

In Theorem 2.2 about the large-time behavior of solutions to (20), the essential quantity $\mathfrak{S}$ has been introduced. Below, we prove that if $\mathfrak{S}(s)$ is negative for some $s>0$, then the $s$-th moment of the solution,

$$
M_{s}(t)=\int_{\mathbb{R}_{+}} v^{s} f(v ; t) d v
$$

remains bounded for all times. On the other hand, if $\mathfrak{S}(s)$ is positive for some $s \geq 2$, then $M_{s}(t)$ diverges exponentially fast as $t \rightarrow \infty$. We exploit this information to prove existence and decay properties of the steady state. 
To start with, we note that conservation of the total wealth allows to conclude that at least all moments of order $s \leq 1$ remain uniformly bounded. In fact, by Hölder's inequality,

$$
\int_{\mathbb{R}_{+}} v^{s} f(v) d v \leq\left(\int_{\mathbb{R}_{+}} v f(v) d v\right)^{s} \cdot\left(\int_{\mathbb{R}_{+}} f(v) d v\right)^{1-s}=M^{s} \quad(0<s<1) .
$$

Now, let $s>1$ and suppose that the initial density $f_{0}(v)$ satisfies

$$
M_{s}(0)=\int_{\mathbb{R}_{+}} v^{s} f_{0}(v) d v<\infty
$$

Then, putting $\Phi(v)=v^{s}$ in the weak form (20), we obtain

$$
\frac{d}{d t} \int_{\mathbb{R}_{+}} v^{s} f(v, t) d v=\frac{1}{2}\left\langle\int_{\mathbb{R}_{+}^{2}} d v d w\left(\sum_{i=1}^{2}\left(p_{i} v+q_{i} w\right)^{s}-v^{s}-w^{s}\right) f(v) f(w)\right\rangle_{+} .
$$

In the following, we establish upper and lower bounds for the right-hand side of (35). These bounds rely on the following elementary inequality:

Lemma 3.1 For arbitrary non-negative real numbers $a$ and $b$, and $s>1$,

$$
\begin{aligned}
& a^{s}+b^{s}+\theta_{s}\left(a^{s-1} b+a b^{s-1}\right) \leq(a+b)^{s} \leq a^{s}+b^{s}+\Theta_{s}\left(a^{s-1} b+a b^{s-1}\right), \\
& \text { with } \theta_{s}=\left\{\begin{array}{ll}
s & (s>3) \\
2^{s-3} s & (2 \leq s \leq 3) \\
0 & (1<s<2)
\end{array} \text { and } \quad \Theta_{s}= \begin{cases}s & (2 \leq s \leq 3) \\
2^{s-3} s & \text { (otherwise) }\end{cases} \right.
\end{aligned}
$$

Remark: An investigation of the limit behavior as $a \searrow 0$ and $b>0$ makes clear that $\theta_{s}=0$ for $1<s<2$ cannot be improved in general.

Proof By homogeneity, it suffices to prove the inequality for $a+b=1$. Define for $s>1$,

$$
\phi(s):=a^{s}+b^{s}+s a b
$$

A calculation yields $\phi(2)=\phi(3)=1$, independently of $a$ and $b=1-a$. Furthermore, $\phi$ is convex in $s$ since

$$
\phi^{\prime \prime}(s)=a^{s} \ln ^{2} a+b^{s} \ln ^{2} b \geq 0 .
$$

Hence $\phi(s) \leq 1$ if and only if $2 \leq s \leq 3$. Observe that $a^{s-2}+b^{s-2}$ is concave w.r.t. $a=1-b \in(0,1)$ for $2 \leq s \leq 3$, and convex for all other $s>1$; the expression attains its extremal value $2^{3-s}$ at $a=b=\frac{1}{2}$. Hence

$$
a^{s-1} b+a b^{s-1}=a b\left(a^{s-2}+b^{s-2}\right) \begin{cases}\leq 2^{3-s} a b & (2 \leq s \leq 3) \\ \geq 2^{3-s} a b & \text { (otherwise) }\end{cases}
$$

Thus we obtain, for $2 \leq s \leq 3$

$$
a^{s}+b^{s}+2^{s-3} s\left(a^{s-1} b+a b^{s-1}\right) \leq \phi(s) \leq 1=(a+b)^{s},
$$


and with reversed inequalities for $1<s<2$ or $s>3$.

Now let $s>1$ be fixed and consider for $a \in[0,1]$

$$
f_{s}(a):=a^{s}+b^{s}+s\left(a^{s-1} b+a b^{s-1}\right),
$$

with $b=1-a$. Observe that $f_{s}(0)=f_{s}(1)=1$, and furthermore

$$
f_{s}^{\prime}(a)=s(s-1)\left(a^{s-2} b-a b^{s-2}\right),
$$

so that $f$ has $\bar{a}=\frac{1}{2}$ as the only critical point in $(0,1)$. For $s<3$, one has $f_{s}(\bar{a})=(s+1) 2^{1-s}>1$, so $\bar{a}$ is a maximum point and hence $f_{s}(a) \geq 1$ for $a \in[0,1]$; for $s>3$, it is a minimum point and $f_{s}(a) \leq 1$. Consequently, for $s \leq 3$,

$$
a^{s}+b^{s}+s\left(a^{s-1} b+a b^{s-1}\right)=f_{s}(a) \geq 1=(a+b)^{s} ;
$$

the reversed inequality holds for $s \geq 3$.

Using the upper bound in (36), estimate

$$
\left(p_{i} v+q_{i} w\right)^{s} \leq p_{i}^{s} v^{s}+q_{i}^{s} w^{s}+\Theta_{s}\left(p_{i}^{s-1} q_{i} v^{s-1} w+p_{i} q_{i}^{s-1} v w^{s-1}\right)
$$

under the integral in (35), leading to

$$
\begin{aligned}
\frac{d}{d t} M_{s}(t) \leq & \frac{1}{2}\left\langle\int_{\mathbb{R}_{+}^{2}} d v d w\left(\left(p_{1}^{s}+p_{2}^{s}-1\right) v^{s}+\left(q_{1}^{s}+q_{2}^{s}-1\right) w^{s}\right) f(v) f(w)\right\rangle \\
& +\frac{1}{2} \Theta_{s}\left\langle\int_{\mathbb{R}_{+}^{2}} d v d w\left(\left(p_{1}^{s-1} q_{1}+p_{2}^{s-1} q_{2}\right) v^{s-1} w+\left(p_{1} q_{1}^{s-1}+p_{2} q_{2}^{s-1}\right) v w^{s-1}\right) f(v) f(w)\right\rangle \\
= & S(s) \cdot \int_{\mathbb{R}_{+}} v^{s} f(v) d v+\Theta_{s}\left\langle p_{i}^{s-1} q_{i}+p_{i} q_{i}^{s-1}\right\rangle_{+} \cdot \int_{\mathbb{R}_{+}} v f(v) d v \int_{\mathbb{R}_{+}} w^{s-1} f(w) d w .
\end{aligned}
$$

Recall that the total wealth $\int v f(v) d v=M$ is conserved in time. Further, by Hölder's inequality, it follows that

$$
\int_{\mathbb{R}_{+}} w^{s-1} f(w) d w \leq\left(\int_{\mathbb{R}_{+}} w^{s} f(w) d w\right)^{1-\frac{1}{s}}
$$

Hence, we obtain

$$
\frac{d}{d t} \int_{\mathbb{R}_{+}} v^{s} f(v) d v \leq \mathfrak{S}(s) \int_{\mathbb{R}_{+}} v^{s} f(v) d v+\Theta_{s} \cdot \mathfrak{K}(s) \cdot M \cdot\left(\int_{\mathbb{R}_{+}} v^{s} f(v) d v\right)^{1-\frac{1}{s}},
$$

where

$$
\mathfrak{K}(s):=\left\langle p_{i}^{s-1} q_{i}+p_{i} q_{i}^{s-1}\right\rangle_{+} \leq\left\langle p_{i}^{s}+q_{i}^{s}\right\rangle_{+}=\mathfrak{S}(s)+1 .
$$

In particular, if $\mathfrak{S}(s)$ is a finite number, then so is $\mathfrak{K}(s)$. In this case, the $s$ th moment grows at most exponentially, with rate $\mathfrak{S}(s)$, if it was finite initially. Moreover, if $\mathfrak{S}(s)<0$, then the $s$ thoment 
remains uniformly bounded for all times. In fact, an upper bound on the sth moment is determined by the solution of the associated ordinary differential equation

$$
\dot{y}=\mathfrak{S} \cdot y+\Theta_{s} \cdot \mathfrak{K}(s) \cdot M \cdot y^{1-\frac{1}{s}}
$$

with initial condition $y(0)=M_{s}(0)$. The solution is explicitly given by

$$
y(t)=\left[\left(M_{s} \cdot \exp \{t \cdot \mathfrak{S}(s)\}\right)^{1 / s}+\frac{\Theta_{s} \cdot \mathfrak{K}(s) \cdot M}{\mathfrak{S}(s)}\left(\exp \left\{\frac{t}{s} \cdot \mathfrak{S}(s)\right\}-1\right)\right]^{s} .
$$

Notice that the first term in the square bracket vanishes for $t \rightarrow \infty$ if $\mathfrak{S}(s)<0$, so that the limiting value depends on the initial condition $f_{0}$ only through the total wealth $M$.

By the same reasoning as above, we construct a bound from below on the time-derivative of the integral. For this, we use the lower bound given in the elementary inequality (36). Replacing the respective expressions under the integral, we obtain

$$
\begin{aligned}
\frac{d}{d t} M_{s}(t) \geq & \frac{1}{2} \int_{\mathbb{R}_{+}^{2}} d v d w\left(\left\langle p_{1}^{s}+p_{2}^{s}-1\right\rangle v^{s}+\left\langle q_{1}^{s}+q_{2}^{s}-1\right\rangle w^{s}\right) f(v) f(w) \\
& +\theta_{s}\left\langle\int_{\mathbb{R}_{+}^{2}} d v d w\left(\left(p_{1}^{s-1} q_{1}+p_{2}^{s-1} q_{2}\right) v^{s-1} w+\left(p_{1} q_{1}^{s-1}+p_{2} q_{2}^{s-1}\right) v w^{s-1}\right) f(v) f(w)\right\rangle \\
= & \mathfrak{S}(s) \cdot \int v^{s} f(v, t) d v+\theta_{s} \cdot \mathfrak{K}(s) \cdot \int_{\mathbb{R}_{+}} v f(v) d v \int_{\mathbb{R}_{+}} w^{s-1} f(w) d w .
\end{aligned}
$$

We use Hölder's inequality to estimate

$$
\int_{\mathbb{R}_{+}} w^{s-1} f(w) d w \geq\left(\int_{\mathbb{R}_{+}} w f(w) d w\right)^{s-1}=M^{s-1} .
$$

By Gronwall's inequality, a lower bound is given by

$$
M_{s}(t) \geq M_{s}(0) \cdot \exp \{t \cdot \mathfrak{S}(s)\}+\frac{\theta_{s} \cdot \mathfrak{K}(s) \cdot M^{s}}{\mathfrak{S}(s)}(\exp \{t \cdot \mathfrak{S}(s)\}-1) .
$$

We conclude that if $\mathfrak{S}(s)>0$, then the moment $M_{s}$ diverges exponentially in time.

In the special case that $\mathfrak{S}(s)=0$, similar but simpler arguments give that the corresponding moment remains either bounded (iff $\mathscr{K}(s)=0$ ) or diverges, but only at the algebraic rate $t^{s}$. Finally, if $\mathfrak{S}(s)=+\infty$, an easy argument shows that the $s$ th moment of $f(v, t)$ must be infinite for all positive times.

Theorem 3.2 Consider a solution $f$ to the Boltzmann equation (20). Let $s>1$ be such that $M_{s}(0)=\int_{\mathbb{R}_{+}} v^{s} f_{0}(v) d v<\infty$.

1. If $0<\mathfrak{S}(s)<+\infty$, then the sth moment diverges exponentially fast as $t \rightarrow \infty$ :

$$
M_{s}+\frac{\theta_{s} \mathfrak{K}(s)}{\mathfrak{S}(s)} \cdot M^{s}+o(1) \leq \frac{\int_{\mathbb{R}_{+}} v^{s} f(v, t) d v}{\exp \{t \cdot \mathfrak{S}(s)\}} \leq\left[M_{s}^{1 / s}+\frac{\Theta_{s} \mathfrak{K}(s)}{\mathfrak{S}(s)} \cdot M\right]^{s}+o(1) .
$$


2. If $\mathfrak{S}(s)<0$, then the sth moment remains uniformly bounded as $t \rightarrow \infty$ :

$$
\frac{\theta_{s} \mathfrak{K}(s)}{|\mathfrak{S}(s)|} \cdot M^{s}+o(1) \leq \int_{\mathbb{R}_{+}} v^{s} f(v, t) d v \leq\left(\frac{\Theta_{s} \mathfrak{K}(s)}{|\mathfrak{S}(s)|}\right)^{s} \cdot M^{s}+o(1) .
$$

3. If $\mathfrak{S}(s)=0$, then the sth moment either remains bounded or diverges at an algebraic rate:

$$
\left(\frac{\theta_{s} \mathfrak{K}(s)}{s}\right)^{s} \cdot M^{s}+o(1) \leq t^{-s} \cdot \int_{\mathbb{R}_{+}} v^{s} f(v, t) d v \leq\left(\frac{\Theta_{s} \mathfrak{K}(s)}{s}\right)^{s} \cdot M^{s}+o(1) .
$$

4. Finally, if $\mathfrak{S}(s)=+\infty$, then the sth moment is infinite for all $t>0$.

\subsection{Steady state as the long-time limit}

Below, the unique steady state $f_{\infty}$ to the kinetic equation (20) is obtained as the limit of an arbitrary solution $f(v, t)$ for $t \rightarrow \infty$ in a suitable $d_{s}$-norm. Since the long-time behavior of solutions is essentially determined by the quantity $\mathfrak{S}$, we need to start by investigating this function in further detail.

First recall that for an arbitrary non-negative number $p$, the exponential $s \mapsto p^{s}$ is convex in $s>0$. Hence $\mathfrak{S}(s)$, which is the average of convex functions, is convex on its domain. By the dominated convergence theorem, $\mathfrak{S}(s)$ is well-defined at least for $0<s \leq 1$, but possibly $\mathfrak{S}(s)=+\infty$ for all $s>s_{\infty}>1$. Since $\mathfrak{S}(1)=0$, convexity leaves only three possibilities for the behavior of $\mathfrak{S}$ :

1. $\mathfrak{S}(s)$ is non-negative for all $s>0$.

2. $\mathfrak{S}(s)$ is negative for some $s \in(0,1)$, and positive for all $s>1$.

3. $\mathfrak{S}(s)$ is negative for all $1<s<\bar{s}$, and positive for all $s<1$ and all $s>\bar{s}$; here either $\bar{s}=+\infty$, or $\mathfrak{S}(\bar{s})=0$, or $\mathfrak{S}(s)=+\infty$ for $s>\bar{s}$.

If $\mathfrak{S}$ is differentiable at $s=1$, then the first case corresponds to $\mathfrak{S}^{\prime}(1)=0$, the second to $\mathfrak{S}^{\prime}(1)>0$, and the last to $\mathfrak{S}^{\prime}(1)<0$. These four cases are now discussed in detail.

In the first case, no information about the existence of a long-time limit can be extracted.

In the second case, $r:=-\mathfrak{S}(s)>0$ for some $s \in(0,1)$. Then the solution $f(v, t)$ has the Cauchy-property w.r.t. $t \geq 0$, i.e., for each $\varepsilon>0$, there exists a time $T(\varepsilon)$ such that

$$
d_{s}\left(f\left(t_{1}\right), f\left(t_{2}\right)\right) \leq \varepsilon \text { for all } t_{1}, t_{2} \geq T(\varepsilon) .
$$

To prove this, let $t_{1}>t_{2} \geq 0$ be arbitrary, and write $t_{1}=t_{2}+n \Delta$ with some $\frac{1}{2} \leq \Delta \leq 1$ and $n \in \mathbb{N}$. By the triangle inequality and the contraction estimate from Theorem 2.2,

$$
\begin{aligned}
d_{s}\left(f\left(t_{1}\right), f\left(t_{2}\right)\right) & \leq \sum_{k=0}^{n-1} d_{s}\left(f\left(t_{2}+(k+1) \Delta\right), f\left(t_{2}+k \Delta\right)\right) \\
& \leq \sum_{k=0}^{n-1} e^{-r\left(t_{2}+k \Delta\right)} d_{s}(f(\Delta), f(0)) \\
& \leq \frac{e^{-r t_{2}}}{1-e^{-r \Delta}} d_{s}\left(f(\Delta), f_{0}\right) \leq e^{-r t_{2}} \sup _{1 / 2 \leq \Delta^{\prime} \leq 1} \frac{d_{s}\left(f\left(\Delta^{\prime}\right), f_{0}\right)}{1-e^{-r \Delta^{\prime}}} .
\end{aligned}
$$


As $f(t)$ is continuous in the $d_{s}$-metric, the last supremum is finite, which implies (43). In addition, the first moment of $f(v, t)$ equals $M$ for all $t \geq 0$. The Cauchy property together with this uniform bound on moments is enough to conclude convergence of $f(v, t)$ to a limit $f_{\infty}(v)$ in $d_{s}$ as $t \rightarrow+\infty$, see [8]. This limit is a probability density with first moment less or equal to $M$.

Now let $f^{\prime}(t)$ be another solution to the Boltzmann equation (20), with first moment equal to $M^{\prime}$. Then $f^{\prime}(t)$ converges to a limit $f_{\infty}^{\prime}$ as $t \rightarrow+\infty$, with first moment less or equal to $M^{\prime}$. On the other hand, as two probability measures always have finite $d_{s}$-distance for $s<1$, it follows from Theorem 2.2 that $d_{s}\left(f(t), f^{\prime}(t)\right) \rightarrow 0$ as $t \rightarrow+\infty$. Consequently, $f_{\infty}=f_{\infty}^{\prime}$, and the first moment is less or equal than the minimum of $M$ and $M^{\prime}$.

It follows that for an arbitrary initial distribution $f_{0}$, the solution $f(t)$ converges to the same limit $f_{\infty}$. This limit is a probability density with first moment equal to zero, i.e., it is a Dirac distribution centered at $v=0$. Notice that, although $f_{\infty}$ possesses finite moments of arbitrary order while $\mathfrak{S}(s)>0$ for $s>1$, this is not in contradiction to Theorem 3.2, since also $M=0$.

The third case is the most interesting one. Choose some $s \in(1,2)$ with $s<\bar{s}$; then $r:=$ $-\mathfrak{S}(s)>0$. Assume that the initial datum of $f$ possesses a moment of order $S>s$. By the same argument as above, it follows that $f(v, t)$ has the Cauchy property in $d_{s}$; notice that $d_{s}\left(f(t), f_{0}\right)$ is always finite since $s<2$ and the first moment (mean wealth) is conserved under evolution. Moreover, as we required $s<\bar{s}$, there exists a $s^{\prime}$ with $s<s^{\prime}<\min (\bar{s}, S)$; by Theorem 3.2, the moment of order $s^{\prime}$ remains uniformly bounded. It follows that $f(t)$ converges in $d_{s}$ to a limit distribution $f_{\infty}(v)$, which is normalized and has the same first moment as the $f(t)$.

This convergence implies that $f_{\infty}$ is a steady state for the kinetic equation (20). Indeed, denote by $f_{\infty}(t)$ the solution to (20) with initial datum $f_{\infty}$, then Theorem 2.2 gives

$$
\begin{aligned}
d_{s}\left(f_{\infty}(t), f_{\infty}\right) & \leq d_{s}\left(f_{\infty}(t), f(t+T)\right)+d_{s}\left(f(t+T), f_{\infty}\right) \\
& \leq e^{-r t} d_{s}\left(f_{\infty}, f(T)\right)+d_{s}\left(f(t+T), f_{\infty}\right) .
\end{aligned}
$$

The last expression can be made arbitrarily small by choosing $T$ large enough, so that $f_{\infty}(t)=f_{\infty}$ for all $t \geq 0$. In fact, $f_{\infty}$ is the only steady state with the respective value of the first moment; for if $f_{\infty}^{\prime}$ is another steady state with the same first moment, then $d_{s}\left(f_{\infty}, f_{\infty}^{\prime}\right)$ is finite, and so, invoking Theorem 2.2 again,

$$
d_{s}\left(f_{\infty}, f_{\infty}^{\prime}\right) \leq e^{-r} d_{s}\left(f_{\infty}, f_{\infty}^{\prime}\right)
$$

which forces $f_{\infty}=f_{\infty}^{\prime}$. Finally, consider a solution $f$ which has arbitrary moments bounded initially. Theorem 3.2 gives a time-uniform bound for moments of order less than $\bar{s}$. Since convergence $f(t) \rightarrow f_{\infty}$ in $d_{s}$ implies weak* convergence of the associated measures, it follows that $f_{\infty}$ has finite moments of all orders less than $\bar{s}$. On the other hand, no moment of order larger than $\bar{s}$ can be finite. To see this, simply apply Theorem 3.2 to the steady state solution $f_{\infty}(t) \equiv f_{\infty}$ to derive a contradiction.

We summarize the results of this section:

Theorem 3.3 Let $f(v, t)$ be a weak solution of the Boltzmann equation (20), which has initially finite moments up to order $S>0$. Further assume that $\mathfrak{S}(s)<0$ for some $s \in(0, S)$. Then $f$ converges exponentially fast in $d_{s}$ to a steady state $f_{\infty}$,

$$
d_{s}\left(f(t), f_{\infty}\right) \leq d_{s}\left(f_{0}, f_{\infty}\right) \exp \{-|\mathfrak{S}(s)| t\} .
$$


If $s<1$, then $f_{\infty}$ is a Dirac distribution centered at $v=0$, and there are no other steady states. If $s>1$, then $f_{\infty}$ has mean wealth equal to $M$, and it is the only steady state with this mean wealth. Moreover, if $\mathfrak{S}\left(s^{\prime}\right)<0$ exactly for $1<s^{\prime}<\bar{s}$, possibly with $\bar{s}=+\infty$, then $f_{\infty}$ has finite moments of all orders less than $\bar{s}$, while moments of order larger than $\bar{s}$ are infinite.

Remark 3.4 It is possible that $d_{s}\left(f(t), f_{\infty}\right)$ is infinite in (44); this simply means that also $d_{s}\left(f_{0}, f_{\infty}\right)$ is infinite.

\subsection{Smoothness of the steady state}

Theorem 3.5 Assume that there are positive numbers $r$ and $\delta$ such that

$$
p_{i}^{r}+q_{i}^{r} \geq 1+\delta^{r} \quad \text { a.s. }
$$

Assume further that $\mathfrak{S}(\sigma)<0$ for some $1<\sigma<2$, so that a non-trivial steady state $f_{\infty}$ to the kinetic equation (20) exists. Then either $f_{\infty}$ is a Dirac distribution centered at $v=M$, or $f_{\infty}$ is a smooth function and belongs to the r-th Gevrey class, i.e.

$$
\left|\widehat{f}_{\infty}(\xi)\right| \leq \exp \left(-\mu|\xi|^{r}\right), \quad \text { for }|\xi| \geq \rho,
$$

with suitable positive numbers $\rho$ and $\mu$.

Remark 3.6 Since the mean wealth is preserved, i.e. $\left\langle p_{i}+q_{i}\right\rangle_{+}=1$, it follows that $r<1$. Notice that condition (45) imposes no serious restrictions on the distribution of the interaction coefficients. For instance, it allows any probability measure which is compactly supported in the interior of the first quadrant of the $p_{i}-q_{i}-$ plane.

The proof of Theorem 3.5 is done on the level of Fourier transforms, and is divided into a series of Lemmas. The first is concerned with the behavior of $\widehat{f}_{\infty}$ near the origin:

Lemma 3.7 Under the hypotheses of Theorem 3.5, either $f_{\infty}$ is a Dirac distribution, i.e. $|\widehat{f}| \equiv 1$, or there exist positive numbers $\rho$ and $\kappa$ s.t.

$$
\left|\widehat{f}_{\infty}(\xi)\right| \leq \exp \left(-\kappa \xi^{2}\right), \quad \text { for }|\xi|<\rho .
$$

Proof First assume that $f_{\infty}$ has finite second moment. Then $\widehat{f}_{\infty}$ is twice differentiable at $\xi=0$, and

$$
\widehat{f}_{\infty}(\xi)=1-i M \xi-\frac{1}{2} Q \xi^{2}+o\left(\xi^{2}\right)
$$

where $M$ and $Q$ denote the first and second moment of $f_{\infty}$, respectively. This means that

$$
\left|\widehat{f}_{\infty}(\xi)\right|^{2}=1-\left(Q-M^{2}\right) \xi^{2}+o\left(\xi^{2}\right) .
$$

Now either $Q=M^{2}$, which implies that $f_{\infty}$ is concentrated at $\xi=M$, or $\kappa:=\frac{1}{4}\left(Q-M^{2}\right)>0$ makes (46) true for some small $\rho>0$. 
In the case that the second moment of $\widehat{f}_{\infty}$ is infinite, we write

$$
\widehat{f}_{\infty}(\xi)=\int_{\mathbb{R}} f_{\infty}(v) e^{-i \xi v} d v=1-\underbrace{\int_{\mathbb{R}}(1-\cos (\xi v)) d f_{\infty}(v)}_{=: H_{1}(\xi)}-i \underbrace{\int_{\mathbb{R}} \sin (\xi v) d f_{\infty}(v)}_{=: H_{2}(\xi)},
$$

so that

$$
\left|\widehat{f}_{\infty}(\xi)\right|^{2}=1-2 H_{1}(\xi)+H_{1}(\xi)^{2}+H_{2}(\xi)^{2} .
$$

The elementary inequalities $1-\cos x \leq|x|$ and $|\sin x| \leq|x|$ for $x \in \mathbb{R}$ imply

$$
H_{1}(\xi) \leq|\xi| \int_{\mathbb{R}} v d f_{\infty}(v)=M|\xi|, \quad\left|H_{2}(\xi)\right| \leq|\xi| \int_{\mathbb{R}} v d f_{\infty}(v)=M|\xi| .
$$

On the other hand, with the elementary inequalities

$$
1-\cos x \geq \frac{1}{3} x^{2} \quad \text { for }|x| \leq 1, \quad 1-\cos x \geq 0 \quad \text { for } x \in \mathbb{R},
$$

one obtains for arbitrary $\xi \in \mathbb{R}$

$$
H_{1} \geq \frac{\xi^{2}}{3} \int_{|\xi v|<1} v^{2} d f_{\infty}(v)=A(\xi) \xi^{2} .
$$

Here $A(\xi)$ is a positive, non-increasing function with $\lim _{\xi \rightarrow 0} A(\xi)=+\infty$, since the second moment of $f_{\infty}$ diverges. Choosing $\rho>0$ so small that $A(\rho) \geq 2 M^{2}$, it follows that

$$
\left|\hat{f}_{\infty}(\xi)\right|^{2} \leq 1-2 M^{2} \xi^{2}, \quad \text { for }|\xi|<\rho .
$$

Hence (46) holds with $\kappa:=\frac{1}{2} M^{2}$, possibly after diminishing $\rho$ suitably.

Inspired by the ideas of Bobylev et al in [5], and those of Desvillettes at al in [17], we introduce the fixed point operator

$$
\mathbf{R}[\psi](\xi):=\left\{\begin{array}{cc}
\widehat{f}_{\infty}(\xi) & \text { if }|\xi|<\rho \\
\left\langle\psi\left(p_{i} \xi\right) \psi\left(q_{i} \xi\right)\right\rangle_{+} & \text {if }|\xi| \geq \rho .
\end{array}\right.
$$

on bounded functions $\psi: \mathbb{R} \rightarrow \mathbb{C}$. Notice that $\mathbf{R}$ is closely related to the Fourier transform of the collision kernel. Furthermore, for $\mu>0$, define $K_{\mu}$ as the set of functions $\psi$ with $\psi(0)=1$, $\psi^{\prime}(0)=\widehat{f}_{\infty}^{\prime}(0)$, and satisfying the estimates

$$
|\psi(\xi)| \leq \exp \left(-\kappa \xi^{2}\right) \quad \text { for }|\xi|<\rho, \quad|\psi(\xi)| \leq \exp \left(-\mu|\xi|^{r}\right) \quad \text { for }|\xi| \geq \rho .
$$

Here $q$ and $\rho$ are the constants from Lemma 3.7 above. Eventually, we wish to prove that $\widehat{f}_{\infty} \in K_{\mu}$ for a suitable $\mu>0$.

Lemma 3.8 Under the hypotheses of Theorem 3.5, and assuming that $f_{\infty}$ is not a Dirac distribution, there exists some $\mu>0$, depending only on $\rho$ and $\kappa$, such that $\mathbf{R}$ maps $K_{\mu}$ into itself. 
Proof By definition of $\mathbf{R}$, it is obvious that $\mathbf{R}[\psi](0)=1$ and $\mathbf{R}[\psi]^{\prime}(0)=\widehat{f}_{\infty}^{\prime}(0)$. Moreover, Lemma 3.7 assures that the first estimate in (47) holds. Now let $\psi \in K_{\mu}$ and $\xi \in \mathbb{R}$ with $|\xi| \geq \rho$ be arbitrary. We show that

$$
e^{\mu|\xi|^{r}} \cdot|\mathbf{R}[\psi](\xi)| \leq\left\langle e^{\mu|\xi|^{r}} \cdot\left|\psi\left(p_{i} \xi\right)\right| \cdot\left|\psi\left(q_{i} \xi\right)\right|\right\rangle_{+} \leq 1 .
$$

To this end, we prove that the term inside the expectation value is less/equal one a.s. We distinguish four cases:

1. If both $p_{i}|\xi| \geq \rho$ and $q_{i}|\xi| \geq \rho$, then the hypothesis (45) yields

$$
e^{\mu|\xi|^{r}} \cdot\left|\psi\left(p_{i} \xi\right)\right| \cdot\left|\psi\left(q_{i} \xi\right)\right| \leq e^{\mu|\xi|^{r}-\mu p_{i}^{r}|\xi|^{r}-\mu q_{i}^{r}|\xi|^{r}} \leq e^{-\mu|\xi|^{r} \delta^{r}} \leq 1 .
$$

2. If both $p_{i}|\xi|<\rho$ and $q_{i}|\xi|<\rho$, then we conclude from (45) that $p_{i}^{2}+q_{i}^{2} \geq 2^{1-2 / r}$, so

$$
e^{\mu|\xi|^{r}} \cdot\left|\psi\left(p_{i} \xi\right)\right| \cdot\left|\psi\left(q_{i} \xi\right)\right| \leq e^{\mu|\xi|^{r}-\kappa p_{i}^{2}|\xi|^{2}-\kappa q_{i}^{2}|\xi|^{2}} \leq e^{-|\xi|^{r}\left(\mu-\kappa \cdot 2^{1-2 / r} \rho^{2-r}\right)} \leq 1,
$$

provided that $\mu \leq 2^{1-2 / r} \rho^{2-r} \cdot \kappa$.

3. Now assume that $p_{i}|\xi|<\rho$ while $q_{i}|\xi| \geq \rho$. Using (45) once again, one finds

$$
e^{\mu|\xi|^{r}} \cdot\left|\psi\left(p_{i} \xi\right)\right| \cdot\left|\psi\left(q_{i} \xi\right)\right| \leq e^{\mu|\xi|^{r}-\kappa p_{i}^{2}|\xi|^{2}-\mu q_{i}^{r}|\xi|^{r}} \leq e^{\mu\left(p_{i}^{r}-\delta^{r}\right)|\xi|^{r}-\kappa p_{i}^{2}|\xi|^{2}}
$$

Obviously, the last expression is less than one if $p_{i}<\delta$. Assume now that $p_{i} \geq \delta$. Since $|\xi| \geq \rho$, one has $p_{i}|\xi| \geq \delta \rho$ and hence the exponent can be estimated by

$$
\mu p_{i}^{r}|\xi|^{r}-\kappa p_{i}^{2}|\xi|^{2} \leq p_{i}^{r}|\xi|^{r}\left(\mu-\kappa \delta^{2-r} \rho^{2-r}\right) .
$$

The last expression is negative provided that $\mu \leq(\delta \rho)^{2-r} \cdot \kappa$.

4. The last case, $p_{i}|\xi| \geq \rho$ and $q_{i}|\xi|<\rho$, is treated in the same way and yields the same condition on $\mu$.

In summary, (48) holds if $\mu$ is the minimum of $2^{1-2 / r} \rho^{2-r} \cdot \kappa$ and $(\delta \rho)^{2-r} \cdot \kappa$.

Lemma 3.9 Under the hypotheses of Theorem 3.5, and assuming that $f_{\infty}$ is not a Dirac distribution, $\widehat{f}_{\infty} \in K_{\mu}$ with the $\mu$ from Lemma 3.8 above.

Proof Let $\psi_{0}(\xi)=\widehat{f}_{\infty}(\xi)$ for $|\xi|<\rho$, and $\psi_{0}(\xi)=0$ for $|\xi| \geq \rho$. Define inductively the sequence $\psi_{n+1}=\mathbf{R}\left[\psi_{n}\right]$. By Lemma 3.8, each $\psi_{n}$ belongs to $K_{\mu}$. Moreover, by the same estimates that lead to Theorem 2.2, one proves that $\mathbf{R}$ acts as a contraction on functions $\psi, \varphi$ with $|\psi| \leq 1$, $|\varphi| \leq 1$ and $\psi(0)=\varphi(0)=1, \psi^{\prime}(0)=\varphi^{\prime}(0)=\widehat{f}_{\infty}^{\prime}(0)$. In fact, $|\mathbf{R}[\psi](\xi)-\mathbf{R}[\phi](\xi)|=0$ for $|\xi|<\rho$, while for $\xi \geq \rho$, one finds

$$
|\mathbf{R}[\psi](\xi)-\mathbf{R}[\varphi](\xi)| \leq \sup _{\xi^{\prime} \neq 0}\left(\frac{\left|\psi\left(\xi^{\prime}\right)-\varphi\left(\xi^{\prime}\right)\right|}{\left|\xi^{\prime}\right|^{\sigma}}\right) \cdot\left\langle p_{i}^{\sigma}+q_{i}^{\sigma}\right\rangle_{+} \cdot|\xi|^{\sigma} .
$$


The supremum on the right-hand side is finite (recall that $1<\sigma<2$ ). By definition of $K_{\mu}$, inequality (49) applies in particular to any pair $\psi_{n}, \psi_{m}$, yielding

$$
\left|\psi_{n+1}(\xi)-\psi_{n}(\xi)\right| \leq C \cdot\left\langle p_{i}^{\sigma}+q_{i}^{\sigma}\right\rangle_{+}^{n} \cdot|\xi|^{\sigma}
$$

with some finite constant $C$. By hypothesis, $\mathfrak{S}(\sigma)<0$, so $0<\left\langle p_{i}^{\sigma}+q_{i}^{\sigma}\right\rangle_{+}<1$, implying local uniform convergence of the sequence $\psi_{n}$ to a limit function $\psi_{\infty}$. Observe that $\psi_{\infty}$ satisfies the same pointwise estimates (47) as the $\psi_{n}$, i.e. $\psi_{\infty} \in K_{\mu}$. Moreover, $\psi_{\infty}(\xi)=\widehat{f}_{\infty}(\xi)$ for $|\xi|<\rho$. Finally, local uniform convergence in combination with the pointwise estimates (47) is more than enough to invoke Lebesgue's dominated convergence theorem and conclude

$$
\psi_{\infty}(\xi)=\left\langle\psi_{\infty}\left(p_{i} \xi\right) \psi_{\infty}\left(q_{i} \xi\right)\right\rangle_{+}, \quad \text { for }|\xi| \geq \rho,
$$

i.e. $\psi_{\infty}$ is a fixed point of the operator $\mathbf{R}$. By (49), we conclude that $\psi_{\infty}$ is in fact the unique fixed point of $\mathbf{R}$ among the functions $\psi$ with $|\psi| \leq 1, \psi(0)=1$ and $\psi^{\prime}(0)=\widehat{f}_{\infty}^{\prime}(0)$.

Obviously, $\widehat{f}_{\infty}$ is also a fixed point of $\mathbf{R}$, as $(50)$ is nothing but the equation for the stationary state in Fourier representation, i.e. (28) with $\partial_{t} \widehat{f}=0$. So $\widehat{f}_{\infty}=\psi_{\infty} \in K_{\mu}$, which finishes the proof.

By definition of $K_{\mu}$, Theorem 3.5 now follows immediately from Lemma 3.9.

\section{Shape of the tails}

We are eventually in the situation to study the behavior of the steady state $f_{\infty}(v)$ for large values of the wealth $v$.

\subsection{Non-pointwise conservative trades}

In this section we study the formation of tails in models which conserve money only in the mean. For certain probability distributions of the interaction coefficients, these tails are of Pareto type. Recall that the decay behavior of the steady state $f_{\infty}$ is essentially determined by the function

$$
\mathfrak{S}(s)=\frac{1}{2}\left\langle p_{1}^{s}+p_{2}^{s}+q_{1}^{s}+q_{2}^{s}\right\rangle-1 .
$$

Algebraic decay corresponds to $\mathfrak{S}(s)$ being negative on a finite interval $(1, \bar{s})$ with $\bar{s}>1$. In view of (1), we define the Pareto index of the stationary distribution $f_{\infty}$ as the number $\mu>0$ such that all moments of $f_{\infty}$ of order less than $\mu$ are finite, while $f_{\infty}$ possesses no moments of order larger than $\mu$. From Theorem 3.3, it is immediate to conclude that the Pareto index $\mu$ coincides with the non-trivial zero $\bar{s}>1$ of $\mathfrak{S}$.

We focus on various special cases of the trade given by the model (15) in the following. The saving propensity $0<\lambda<1$ is fixed, and we assume that the probability variables $\eta$ and $\tilde{\eta}$ are independent and identically distributed. Under these assumptions,

$$
\mathfrak{S}(s)=\left\langle p_{1}^{s}+p_{2}^{s}\right\rangle-1=(1-\lambda)^{s}-1+\int_{-\lambda}^{+\infty}(\lambda+\omega)^{s} d \rho(\omega),
$$

where $\rho(s)$ is the probability density of $\eta$ and $\tilde{\eta}$ on $[-\lambda,+\infty)$. 

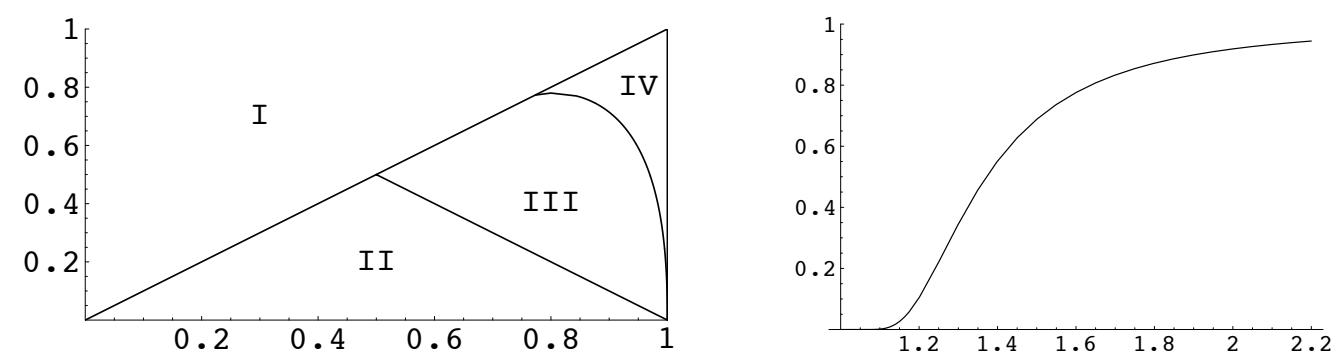

Figure 1: Left Regions for the parameter $\mu$ and saving rate $\lambda$ in Example 2. Pareto Tails are found in zone III. Right Critical value of the saving rate $\lambda$ in Example 4. Pareto tails form for $\lambda$ lying below the curve.

Example 1 If the distribution of $\eta$ is so tame that $\eta \leq \lambda-\delta$ a.s. for some $0<\delta<\lambda$, then

$$
p_{1}, p_{2}, q_{1}, q_{2} \leq 1-\delta \quad \text { a.s. }
$$

Consequently $\mathfrak{S}(s) \leq 2(1-\delta)^{s}-1$, and this expression converges to -1 as $s \rightarrow \infty$. By the basic properties of $\mathfrak{S}$, it follows that $\mathfrak{S}(s)<0$ for all $s>1$. This corresponds to a dynamic in which all moments remain uniformly bounded in time; the steady state has no Pareto tails.

Example 2 Assume $\eta$ only takes values $+\mu$ and $-\mu$, with probability $\frac{1}{2}$ each, where $0 \leq \mu \leq \lambda$. By varying the parameters $\lambda$ and $\mu$, one encounters the whole variety of possible shapes of the tail of $f_{\infty}$. If $\lambda+\mu<1$, then we are back in the trivial scenario (52), where all moments of $f_{\infty}$ are finite. Hence, assume $\lambda+\mu>1$. We calculate

$$
\mathfrak{S}(s)=(1-\lambda)^{s}-1+\frac{1}{2}(\lambda-\mu)^{s}+\frac{1}{2}(\lambda+\mu)^{s} .
$$

The term $(\lambda+\mu)^{s}$ grows exponentially with respect to $s$ and eventually dominates, making the expression (53) positive for large s. We evaluate numerically the derivative

$$
\mathfrak{S}^{\prime}(1)=(1-\lambda) \ln (1-\lambda)+\frac{1}{2}(\lambda-\mu) \ln (\lambda-\mu)+\frac{1}{2}(\lambda+\mu) \ln (\lambda+\mu)
$$

to decide on the structure of the tail of $f_{\infty}$ : a Pareto tail is found for $\mathfrak{S}^{\prime}(1)<0$, the index of which is given by the non-trivial zero $\bar{s}>1$ of $\mathfrak{S}$; for $\mathfrak{S}^{\prime}(1)>0$, the steady state is a Dirac distribution at zero. The result is displayed in figure 1: region II corresponds to slim tails, region III to Pareto tails, and region IV to the singular steady state. Parameter region I is excluded by the condition $\mu \leq \lambda$.

In the following two examples, $\eta$ takes arbitrarily large values.

Example 3 Assume a density with exponential decay,

$$
d \rho(\omega)=\frac{1}{\lambda e} \exp \left(-\frac{\omega}{\lambda}\right) \mathbf{1}_{(-\lambda,+\infty)}(\omega) d \omega .
$$


The integral in (51) converges for all $s>0$. One obtains

$$
\mathfrak{S}(s)=(1-\lambda)^{s}-1+\lambda^{s} \cdot \int_{0}^{\infty} t^{s} e^{-t} d t=(1-\lambda)^{s}-1+\lambda^{s} \cdot \Gamma(1+s) .
$$

The Gamma function grows faster than any exponential, so it eventually dominates the expression, causing high moments to diverge in time. The derivative at $s=1$ reads

$$
\frac{d}{d s} \mathfrak{S}(1)=(1-\lambda) \ln (1-\lambda)+\lambda \ln \lambda+(1-\gamma) \lambda
$$

Here $\gamma=0.577 \ldots$ is Euler's constant. The expression (55) is strictly convex in $\lambda \in[0,1]$, vanishes at $\lambda=0$, and has a unique positive root $\lambda_{0}=0.877 \ldots$ Consequently, Pareto tails form for saving rates $\lambda<\lambda_{0}$.

Example 4 Finally, take $p>1$ and define

$$
d \rho(\omega)=\frac{1}{p} \lambda^{-p}\left(1-\frac{1}{p}\right)^{p} \cdot(\lambda+\omega)^{-(p+1)} \mathbf{1}_{(-\lambda / p,+\infty)}(\omega) d \omega .
$$

It is clear that the integral in (51) diverges to $+\infty$ when $s \nearrow p$, so that at least the moments higher than $p$ become infinite in the long-time limit. Hence $p$ constitutes an upper bound for the Pareto index. For $s<p$,

$$
\mathfrak{S}(s)=(1-\lambda)^{s}-1+\frac{p}{p-s}\left(1-\frac{1}{p}\right)^{s} \lambda^{s}
$$

Numerical investigations indicate that Pareto tails form for $\lambda<\lambda_{p}$, with $\lambda_{p} \approx 1$ for $p>2$. In Figure 1, the values of $\lambda_{p}$ are displayed for $p<2$.

\subsection{Pointwise conservative trades and their nonlinear generalizations}

The main result about pointwise conservative trades - satisfying (5) and (7) - is that they cannot form Pareto tails in the long-time limit.

Theorem 4.1 For each trade of type (5) which is pointwise conservative (7), exactly one of the following is true:

1. Any solution $f$ converges exponentially fast (in any $d_{s}$ with $s>1$ ) to the unique steady state $f_{\infty}$, which possesses finite moments of all orders.

2. Any solution $f$ is constant in time, i.e. $f(t, v)=f_{0}(v)$ for all $t>0$.

3. Along any solution $f$, all moments above the first diverge at an algebraic rate, and the unique stationary state is given by the Dirac distribution centered at $v=0$.

Proof From the behavior of the functions $s \mapsto p^{s}+(1-p)^{s}$ with $0 \leq p \leq 1$ one easily concludes that $\mathfrak{S}(s)$ is well-defined and non-positive for all $s \geq 1$. Furthermore, $\mathfrak{S}(s)$ is zero for some $s>1$ exactly if it is identically zero for all $s$. 
The first case in the Theorem is the generic one, where $\mathfrak{S}$ is not identically zero. The claim follows immediately from Theorem 3.3.

If $\mathfrak{S} \equiv 0$, then $p_{1} p_{2}=q_{1} q_{2}=0$ a.s. Consider the quantity $\mathscr{K}$ defined in (39). This quantity is non-negative. Moreover, it is zero for some $s>1$ exactly if it is zero for all $s$, and the latter is the case if (almost surely) one of the following is true:

$$
p_{1}=q_{2}=1 \text { and } p_{2}=q_{1}=0 \text {, or } p_{1}=q_{2}=0 \text { and } p_{2}=q_{1}=1 .
$$

The corresponding dynamics is trivial, i.e. $f(v, t)=f(v)$ for all $t \geq 0$. If (56) does not hold, then $\mathscr{K}(s)$ is positive for all $s>1$. By Theorem 3.2, all moments of $f$ above the first diverge at algebraic rate. The only possible steady state is the Dirac distribution at $v=0$.

The following three examples illustrate the three cases of qualitative behavior.

Example 5 For a fixed positive number $\lambda>0$, consider the special (deterministic) case of the trade (15)

$$
v^{*}=\lambda v+(1-\lambda) w, \quad w^{*}=(1-\lambda) v+\lambda w .
$$

Each agent possesses the same global saving propensity $\lambda$. It is easily checked that $\mathfrak{S}(s)<0$ for all $s>1$, so the associated steady state $f_{\infty}$ possesses a thin tail, for which all moments are finite. Actually, more can be said: With this radical choice, the model describes a granular gas, for which the Dirac distribution is known to constitute the steady state. Using $\Phi(v)=v^{2}$ as test function in the weak formulation (20), one learns that the variance $V(t)$ of $f(v, t)$ obeys

$$
\frac{d}{d t} V(t)=-2 \lambda(1-\lambda) V(t)
$$

and thus decays to zero at rate $2 \lambda(1-\lambda)$, The solution $f(v, t)$ concentrates in a Dirac distribution centered at $v=M$, the (conserved) mean wealth. In the long-time limit, all agents end up with the same amount of money.

Example 6 Consider the limit cases $\lambda=1$ and $\lambda=0$ in example 5. These correspond to no trading and pure exchange, respectively. One calculates that both $\mathfrak{S}$ and $\mathfrak{K}$ are identically zero here. Note that also the estimate (57) becomes void. These are situations where any probability distribution $f(v)$ constitutes a stationary solution of equation (20).

Example 7 Finally, consider the "Winner takes all" dynamics,

$$
v^{*}=v+w, \quad w^{*}=0
$$

where in every interaction, one agent loses all of his money to the other one. Again, one finds that $\mathfrak{S}$ is identically zero, but now $\mathfrak{K}$ is identically one. Using the indicator function of $[0, \varepsilon]$ in the weak formulation (20), we find

$$
\begin{aligned}
\frac{d}{d t} m_{\varepsilon} & =\frac{1}{2} \iint_{v+w \leq \varepsilon} f(v) f(w) d v d w+\frac{1}{2} \iint_{v, w \geq 0} f(v) f(w) d v d w-m_{\varepsilon} \\
& \geq \frac{1}{2}-m_{\varepsilon}
\end{aligned}
$$


where $m_{\varepsilon}=\int_{0}^{\varepsilon} f(v) d v$ is the mass in the considered interval. Hence $m_{\varepsilon}(t) \geq \frac{1}{2}\left(1-e^{-t}\right) m_{\varepsilon}(0)$, independently of $\varepsilon>0$. In conclusion, mass concentrates at the point $v=0$. The following formal argument indicates the rate at which this concentration occurs: pass to the limit $\varepsilon \rightarrow 0$ in (58), in which $m_{0}(t)$ denotes the mass concentrated in $v=0$ at time $t \geq 0$ (the fraction of truely pauper agents). Then

$$
\frac{d}{d t} m_{0}(t)=\frac{1}{2}\left(1-m_{0}(t)\right)^{2}
$$

Assuming $m_{0}(0)=0$, the solution to this differential equation is $m_{0}(t)=t /(t+2)$, which converges to one as $t \rightarrow \infty$. At the same time, the mean wealth $M$ is conserved; Hölder's inequality implies divergence of all moments above the first, exponentially fast in time. While an increasing majority of agents possesses no money at all, the small fraction of $2 /(t+2)$ agents has become incredibly rich at time $t>0$.

In the pertinent literature, a variety of pointwise conservative trades is proposed, which are not of the type considered here. Such generalized pointwise conservative trades are defined as binary interactions (5) where the sum of the wealth of the agents is maintained. In contrast to the models considered up to here, the degree of randomness is more general in these models. The probability measure according to which the interaction coefficients $p_{i}$ and $q_{i}$ are distributed may depend on the wealth $v, w$ of the interacting agents, and also (in a very sophisticated manner) on time. One example is the minimum exchange model, where $p_{i}$ and $q_{i}$ are non-linear functions of $v / w$. Even more complicated is the model with "personalized" saving propensities from [12]; here the time-dependent probability measure for the interaction coefficients evolves with the solution. (This kinetic model is more conveniently posed on an extended phase space, see [37].) Formally, however, these models can still be written in the form of a weak Boltzmann equation,

$$
\frac{d}{d t} \int \Phi(v) d f(v)=\frac{1}{2} \iint\left(\left\langle\Phi\left(p_{1} v+q_{1} w\right)+\Phi\left(p_{2} v+q_{2} w\right)\right\rangle_{*}-\Phi(v)-\Phi(w)\right) d f(v) d f(w),
$$

where $\langle\cdot\rangle_{*}$ denotes integration against the probability measure, and one has $p_{1}+p_{2}=q_{1}=q_{2}=$ 1 almost surely. The essential difference to (20) is that the two appearing integrations do not commute. Proving existence and uniqueness of solutions $f(v, t)$ to such kinetic models is beyond the scope of this paper. We shall simply assume that a solution $f(v, t)$ of $(60)$ is given.

Theorem 4.2 Assume that $f$ is one solution to a generalized pointwise conservative trade. Then the first moment of $f$ is conserved, and higher moments diverge not faster than algebraically. In fact, if the trade rules are such that there exists $a \delta>0$ for which

$$
\min \left(v^{*}, w^{*}\right) \geq \delta \cdot w,
$$

then all moments of $f$ remain uniformly bounded in time, provided they are finite initially.

Condition (61) expresses that the second agent always saves a tiny portion of his money when trading, and that at least a minimal fraction is passed to the first agent. Solutions to a generalized 
pointwise conservative trade which obey this sensible rule do not develop Pareto tails in the longtime limit. All pointwise conservative trade which do create fat tails for large $t$ can only do so because they violate (61).

Proof This is an extension of the proof for the upper bounds in Theorem 3.2, so we only sketch the necessary changes in the argument. Since $p_{1}+p_{2}=q_{1}+q_{2}=1$ almost surely, one has in the pointwise estimate under the integral in (37)

$$
p_{1}^{s}+p_{2}^{s}-1 \leq 0, \quad q_{1}^{s}+q_{2}^{s}-1 \leq 0, \quad p_{1}^{s-1} q_{1}+p_{1} q_{1}^{s-1}+p_{2}^{s-1} q_{2}+p_{2} q_{2}^{s-1} \leq 2
$$

for all $s>1$. This is enough to conclude, with exactly the same reasoning, algebraic bounds on the divergence of the moments. If moreover (61) holds, then $\delta \leq q_{i} \leq 1-\delta$ for $i=1,2$, leading to $q_{1}^{s}+q_{2}^{s}-1 \leq \delta^{s}+(1-\delta)^{s}<1$ a.s., and thus to uniform boundedness of all moments.

Example 8 Consider model (14) with quenched saving propensities, and assume for simplicity that $\varepsilon \equiv \frac{1}{2}$,

$$
v^{*}=\frac{1}{2}\left(1+\lambda_{A}\right) v+\frac{1}{2}\left(1-\lambda_{B}\right) w, \quad w^{*}=\frac{1}{2}\left(1-\lambda_{A}\right) v+\frac{1}{2}\left(1+\lambda_{B}\right) w .
$$

The individual saving propensities $\lambda_{i}$ are drawn from the interval $[0,1]$ according to a given probability measure $P$. If $\lambda<1-2 \delta$ holds $P$-almost surely, then (61) is satisfied, since then $v^{*} \geq \delta w$ and $w^{*} \geq \frac{1}{2} w$, and the tail of the wealth distribution remains thin for all times.

On the other hand, allowing saving propensities arbitrarily close to one, the distribution $f(v, t)$ may develop a Pareto tail for $t \rightarrow \infty$, see [12] (rather slowly, since this happens on the algebraic time scale). In fact, there is an intimate relation between the density of the probability measure $P$ near $\lambda=0$ and the shape of the tail of $f$ for large times; basically, tails of arbitrary shape can be produced by choosing $P$ appropriately. This has been formally observed in [38], and the convergence of $f$ will be investigated in detail in [37].

Observe that any small perturbation of the model, i.e., any small change of the probability distribution $P$ near $\lambda=0$, will change the long-time behavior of the solutions $f$ completely. The Pareto behavior is structurally unstable.

Example 9 A possible generalized pointwise conservative trade with non-constant transaction coefficients is defined by

$$
v^{*}=v+\psi(v / w) \cdot w, \quad w^{*}=(1-\psi(v / w)) \cdot w,
$$

where $\psi: \mathbb{R}_{+} \rightarrow \mathbb{R}$ is a given continuous non-linear function satisfying the obvious constraint $0 \leq \psi \leq 1$. Independent of the actual shape of $\psi$, the solution $f(v, t)$ to the corresponding kinetic equation will have uniformly bounded moments of all orders if additionally $\delta \leq \psi \leq 1-\delta$. A trivial example that satisfies this condition is the full exchange model with $\psi \equiv \frac{1}{2}$.

An example which does not satisfy this requirement is provided by the minimum exchange model where $\psi(r)=\frac{1}{2} \min (1, r)$, or by the asymmetric exchange model [46], which interpolates between full and minimum exchange. For the latter, $\psi(r)=\frac{1}{2} \min (1,1-\tau(1-r))$ with $\tau \in(0,1)$, and Pareto tails are observed for $\tau \approx 1$ in numerical simulations. 


\subsection{Shape of thin tails}

Finally, we investigate in more detail the situation where

$$
\mathfrak{S}(s)<0 \text { for all } s>1 \text {. }
$$

Here all initially finite moments of the wealth distribution remain uniformly bounded in time, so that the steady state $f_{\infty}$ has finite moments of all orders. Still, there is no information available on the actual shape of the "slim" tail of $f_{\infty}(v)$, apart for that fact that the associated law falls off faster than any negative power of $v$ as $v \rightarrow+\infty$. Below, we investigate in more detail the evolution of moments, and obtain a criterion under which the tail is exponentially small.

The evolution of the principal moments $M_{n}(t)$, with $2 \leq n \in \mathbb{N}$, is determined by

$$
\frac{d}{d t} M_{n}(t)=\left\langle\left(p_{i}^{n}+p_{i}^{n}-1\right)\right\rangle_{+} \cdot M_{n}(t)+\sum_{k=1}^{n-1}\left(\begin{array}{l}
n \\
k
\end{array}\right)\left\langle p_{i}^{k} q_{i}^{n-k}\right\rangle_{+} \cdot M_{k}(t) M_{n-k}(t) .
$$

Taking into account that the first moment is conserved, $M_{1}(t)=M$, equation (63) allows for a recursive computation of the principal moments of the stationary solution,

$$
M_{n}=\frac{\sum_{k=1}^{n-1}\left(\begin{array}{l}
n \\
k
\end{array}\right)\left\langle p_{i}^{k} q_{i}^{n-k}\right\rangle_{+} M_{k} M_{n-k}}{1-\left\langle p_{i}^{n}+q_{i}^{n}\right\rangle_{+}}, \quad n=2,3, \ldots
$$

In particular, starting from $M_{1}=M$, one can calculate - one by one - a sequence of bounded constants $c_{n}$ such that

$$
M_{n}=c_{n} M^{n} .
$$

Observe that the denominator in (64) equals $-\mathfrak{S}(n)$, and hence is positive by assumption (62).

Theorem 4.3 Assume (62). Then the steady state $f_{\infty}$ is a Dirac distribution centered at $v=M$ if and only if $p_{1}+q_{1}=p_{2}+q_{2}=1$ almost surely.

Proof $f_{\infty}$ is a Dirac distribution iff its variance is zero, i.e. $M_{2}=M^{2}$. By (64), this means that

$$
1=c_{2}=\frac{\left\langle\left(p_{i}+q_{i}\right)^{2}\right\rangle_{+}-\left\langle p_{i}^{2}+q_{i}^{2}\right\rangle_{+}}{1-\left\langle p_{i}^{2}+q_{i}^{2}\right\rangle_{+}},
$$

which is equivalent to $\left\langle\left(p_{i}+q_{i}\right)^{2}\right\rangle_{+}=1$. Taking into account that the trade is conservative in the mean, i.e. (10) holds, a standard argument yields further equivalence to $p_{i}+q_{i}=1$ for $i=1,2$.

Under the hypotheses of the Theorem, all agents will eventually end up with exactly the same amount of money. In the framework of the model (13) by Chakraborty and Chakrabarthi, $c_{2}=1$ is realized exactly iff $\varepsilon=1 / 2$ with probability one. In the model (15) of Cordier, Pareschi and Toscani, $c_{2}=1$ corresponds exactly to the degenerate situation $\eta \equiv \tilde{\eta} \equiv 0$ almost surely. The corresponding solutions were studied in Example 5.

In the generic case $c_{2}>1$, the variance of the stationary solution is positive, and information on higher moments is needed in order to extract further properties of the steady state. Unfortunately, even if the recursive computation of the moments is explicit, it is quite difficult to extract from (64) their precise growth. We can, however, establish a criterion under which the tail shows exponential decay. 
Theorem 4.4 Let $f_{\infty}(v)$ be the unique stationary solution to equation (20), corresponding to a trade satisfying condition (62). Assume further that for $n \geq \bar{n}$, with a suitable $\bar{n} \in \mathbb{N}$,

$$
\left\langle\sum_{k=0}^{n} p_{i}^{n-k} q_{i}^{k}\right\rangle_{+} \leq 1
$$

Then the tail of $f_{\infty}(v)$ is exponentially small for $v \rightarrow \infty$ in the sense that

$$
\int_{\mathbb{R}_{+}} \exp (\beta v) f_{\infty}(v, t) d v<\infty
$$

for a positive constant $\beta=\beta(\bar{n})$.

Proof Since all moments $M_{n}$ of the steady state are bounded, $M_{n}=c_{n} M^{n}$, it is always possible to estimate the first $\bar{n}-1$ coefficients $c_{n}$ from above as follows:

$$
c_{n} \leq n ! \alpha^{n} \text { for } n<\bar{n} \text {. }
$$

We assume that $\alpha=\alpha(\bar{n})$ is the smallest possible constant. Then, for all $n<\bar{n}$,

$$
M_{n} \leq n !(\alpha M)^{n}
$$

Bringing together the relations (66) and (67), we obtain from (64)

$$
\begin{aligned}
M_{\bar{n}} & =\frac{\sum_{k=1}^{\bar{n}-1}\left(\begin{array}{l}
\bar{n} \\
k
\end{array}\right)\left\langle p_{i}^{k} q_{i}^{\bar{n}-k}\right\rangle_{+} M_{k} M_{\bar{n}-k}}{1-\left\langle p_{i}^{\bar{n}} q_{i}^{\bar{n}}\right\rangle^{\bar{n}}} \\
& \leq \bar{n} !(\alpha M)^{\bar{n}} \frac{\sum_{k=1}^{\bar{n}-1}\left\langle p_{i}^{k} q_{i}^{\bar{n}-k}\right\rangle_{+}}{1-\left\langle p_{i}^{\bar{n}}+q_{i}^{\bar{n}}\right\rangle_{+}} \\
& =\bar{n} !(\alpha M)^{\bar{n}} \frac{\sum_{k=0}^{\bar{n}}\left\langle p_{i}^{k} q_{i}^{\bar{n}-k}\right\rangle_{+}-\left\langle p_{i}^{\bar{n}}+q_{i}^{\bar{n}}\right\rangle_{+}}{1-\left\langle p_{i}^{\bar{n}}+q_{i}^{\bar{n}}\right\rangle_{+}} \leq \bar{n} !(\alpha M)^{\bar{n}} .
\end{aligned}
$$

Thus condition (67) holds for $n=\bar{n}$. By induction, we conclude (67) for all $n \geq 1$. Hence, for arbitrary positive $\beta<1 /(\alpha M)$, and for any $N \geq 1$,

$$
\int_{0}^{+\infty} \sum_{n=0}^{N} \frac{(\beta v)^{n}}{n !} f_{\infty}(v) d v=\sum_{n=0}^{N} \frac{\beta^{n}}{n !} M_{n} \leq \sum_{n=0}^{N}(\beta \alpha M)^{n} \leq \frac{1}{1-\beta \alpha M}<\infty .
$$

An application of the monotone convergence theorem finishes the proof:

$$
\int_{0}^{+\infty} \exp (\beta v) f_{\infty}(v) d v=\lim _{N \rightarrow \infty} \int_{0}^{+\infty} \sum_{n=0}^{N} \frac{(\beta v)^{n}}{n !} f_{\infty}(v) d v<\infty .
$$

Under the hypotheses of the Theorem, the steady state decays at least as rapidly as a Gibbs distribution

$$
g(v)=\frac{1}{(\alpha M)} \exp \{-v /(\alpha M)\}
$$


This decay behavior is typical for the steady state corresponding to a non realistic pointwise conservative trade with Jacobian equal to 1 . We investigate under which additional assumptions condition (66) is satisfied for the trades (13) of A. Chakraborty and B.K. Chakrabarthi, and (15) of S. Cordier, L. Pareschi and G. Toscani, respectively.

Recall that in (13),

$$
p_{1}-q_{1}=q_{2}-p_{2}=\lambda
$$

which implies $p_{1}>q_{1}$ and $q_{2}>p_{2}$. Therefore

$$
\left\langle\sum_{k=0}^{n} p_{1}^{k} q_{1}^{n-k}\right\rangle=\left\langle\frac{p_{1}^{n+1}-q_{1}^{n+1}}{p_{1}-q_{1}}\right\rangle=\frac{\left\langle p_{1}^{n+1}\left(1-\left(q_{1} / p_{1}\right)^{n+1}\right)\right\rangle}{\lambda} \leq \frac{1}{\lambda}\left\langle p_{1}^{n+1}\right\rangle .
$$

In analogous way, we find

$$
\left\langle\sum_{k=0}^{n} p_{2}^{k} q_{2}^{n-k}\right\rangle \leq \frac{1}{\lambda}\left\langle q_{2}^{n+1}\right\rangle
$$

In order to verify condition (66) it will be enough to prove that, for $n \geq \bar{n}$ sufficiently large, both $\left\langle p_{1}^{n+1}\right\rangle$ and $\left\langle q_{2}^{n+1}\right\rangle$ are less than $\lambda$. Assume the random fraction $\varepsilon$ is absolutely continuous w.r.t. Lebesgue measure $d \varepsilon$, and let $g_{\lambda}(p) d p$ be the corresponding measure for $p_{1}$. Then, there is a $b_{\lambda} \in(\lambda, 1)$ such that

$$
\int_{b_{\lambda}}^{1} g_{\lambda}(p) d p \leq \frac{\lambda}{2}
$$

On the other hand,

$$
\int_{\lambda}^{b_{\lambda}} p^{n} g_{\lambda}(p) d p \leq b_{\lambda}^{n}
$$

Now choose $\bar{n}$ large enough so that $b_{\lambda}^{n_{0}} \leq \lambda / 2$. Then, for all $n \geq \bar{n}$,

$$
\left\langle p_{1}^{n}\right\rangle=\int_{\lambda}^{1} p^{n} g_{\lambda}(p) d p \leq b_{\lambda}^{n}+\frac{\lambda}{2} \leq \lambda .
$$

The previous argument allows to choose the exponent $n_{0}$ (and hence the constant $\alpha$ ) the smaller, the higher the saving rate $\lambda$ is.

Corollary 4.5 Assume that the random fraction $\varepsilon$ in the pointwise conservative trade (13) possesses a Lebesgue integrable density function. Then condition (66) holds, and the stationary state has an exponentially small tail.

Example 10 It is worthwhile to investigate the particular case in which $\varepsilon$ is uniformly distributed on the unit interval. By the considerations above,

$$
\left\langle\sum_{k=0}^{n} p_{1}^{k} q_{1}^{n-k}\right\rangle=\frac{(1-\lambda)^{n+2}+1-\lambda^{n+2}}{(n+2) \lambda(1-\lambda)} .
$$

The latter expression is smaller than one exactly if

$$
\phi_{n}(\lambda):=\frac{1}{n+2}\left[(1-\lambda)^{n+2}+1-\lambda^{n+2}\right]-\lambda(1-\lambda) \leq 0 .
$$


Obviously, $\phi_{n}(1)=0$ and $\phi_{n}(0)=2 /(n+2)>0$. A brief inspection of $\phi_{n}^{\prime}$ reveals that $\phi_{n}$ is monotonically decreasing in $\left[0, \frac{1}{2}\right]$ and monotonically increasing in $\left[\frac{1}{2}, 1\right]$. By elementary by tedious estimates, $\phi_{n}(3 /(n+2)) \leq 0$ for $n=1,2, \ldots$. In conclusion, $\phi_{n}(\lambda) \leq 0$ for $\lambda \in(3 /(n+2), 1)$. One hence finds that $\bar{n} \geq 3 / \lambda-2$ is a possible choice in (66). In particular, $\bar{n}=2$ is possible if $\frac{3}{4} \leq \lambda<1$, in which case $\alpha=1$ in (67). The corresponding steady state $f_{\infty}$ of the economic model decays at least as fast as $\frac{1}{M} \exp (-v / M)$.

We turn to investigate the trade (15). As discussed in Subsection 5.1, for $\eta$ and $\tilde{\eta}$ distributed on the interval $[-\mu,+\mu]$, where $0<\mu \leq \lambda$, for $\mu+\lambda \leq 1$, we are back in the trivial situation (52) which ensures boundedness of all moments. We prove property (66) under the assumption that $\lambda<1$ and that $\eta$ has a Lebesgue integrable density function. By the same argument that lead to Corollary 4.5 , there is a (smallest) integer $n_{0}$ such that

$$
\left\langle(\lambda+\eta)^{k}\right\rangle \leq \frac{\lambda}{2} \text { for all } k \geq n_{0}
$$

Define further $\bar{n} \geq n_{0}$ as the smallest integer satisfying

$$
(1-\lambda)^{\bar{n}-n_{0}} \leq \frac{\lambda}{2}
$$

Then, for all $n \geq \bar{n}$, one has

$$
\begin{aligned}
\left\langle\sum_{k=0}^{n} p_{1}^{k} q_{1}^{n-k}\right\rangle & =\sum_{k=0}^{n}\left\langle(\lambda+\eta)^{k}\right\rangle(1-\lambda)^{n-k} \\
& \leq \sum_{k=0}^{n_{0}}(1-\lambda)^{n-k}+\sum_{k=n_{0}}^{n} \frac{\lambda}{2}(1-\lambda)^{n-k} \\
& \leq(1-\lambda)^{n-n_{0}} \sum_{k=0}^{n_{0}}(1-\lambda)^{k}+\frac{\lambda}{2} \sum_{k=0}^{n-n_{0}}(1-\lambda)^{k} \leq \frac{\lambda}{2} \cdot \frac{1}{\lambda}+\frac{\lambda}{2} \cdot \frac{1}{\lambda}=1 .
\end{aligned}
$$

Corollary 4.6 Assume that the random variables $\eta$ and $\tilde{\eta}$ in the conservative trade (15) are centered and distributed on an interval $[-\mu,+\mu]$, where $0<\mu \leq \lambda$, and $\mu+\lambda \leq 1$, with a Lebesgue integrable density function. Then condition (66) holds, and the stationary state has an exponentially small tail.

Example 11 Again, we provide an explicit calculation that leads to an estimate on the index $\bar{n}$. Namely, assume that $\eta$ and $\tilde{\eta}$ are uniformly distributed in the interval $[-\mu,+\mu]$. For definiteness, assume further that $\frac{1}{2}<\lambda<1$ (the case $0<\lambda<\frac{1}{2}$ is analogous). Then

$$
\begin{aligned}
\left\langle\sum_{k=0}^{n} p_{1}^{k} q_{1}^{n-k}\right\rangle & =\sum_{k=0}^{n}(1-\lambda)^{n-k} \int_{-\mu}^{+\mu}(\lambda+\eta)^{k} \frac{d \eta}{2 \mu} \\
& =\sum_{k=0}^{n}(1-\lambda)^{n-k} \frac{(\lambda+\mu)^{k+1}-(\lambda-\mu)^{k+1}}{2 \mu(k+1)} \\
& \leq \sum_{k=0}^{n}(1-\lambda)^{n-k}(\lambda+\mu)^{k} \leq \frac{(\lambda+\mu)^{n+1}}{(\lambda+\mu)-(1-\lambda)} .
\end{aligned}
$$


The last expression is smaller than one for all $n \geq \bar{n}$ where

$$
\bar{n}:=\frac{\log ((\lambda+\mu)-(1-\lambda))}{\log (\lambda+\mu)}-1 \text {. }
$$

A situation of special interest is where one may choose $\bar{n}=2$, because then $\alpha=1$ is allowed in (67), and the steady state decays at least like $M^{-1} \exp (v / M)$. The corresponding sufficient condition in the regime $\lambda \geq \frac{1}{2}$ can be solved w.r.t. $\mu$, and is equivalent to the inequality

$$
\mu \leq \frac{1}{2}\left(\sqrt{1+6 \lambda-3 \lambda^{2}}-(1+\lambda)\right) .
$$

\section{Conclusions}

We investigated the large-time behavior of various kinetic models describing the fluctuation of wealth in conservative economies. The existence of a unique stationary wealth distribution has been proven. It has been shown that the shape of the tail of this stationary distribution depends on the details of the microscopic interaction. More specifically, we have found that both randomness, e.g. in the form of risky investments, and the agents' propensity to save money play a crucial role in the formation of Pareto tails. In particular, Pareto tails are not present in pointwise conservative models, but appear in models which are conservative in the mean only, when the effects of randomness and saving are well-balanced. Our analysis has been based on the possibility to apply Fourier transform techniques, and it is reminiscent of previous results for the Boltzmann equation for Maxwell molecules [1, 2]. In order to simplify the analysis, we have restricted ourselves to trades where the random distribution (collision kernel) was independent of the wealth of the interacting agents. Specific econophysical models with more complicated collision kernels will be studied in future work.

Acknowledgment: The authors acknowledge support from the Italian MIUR, project "Kinetic and hydrodynamic equations of complex collisional systems". Daniel Matthes thanks the Department of Mathematics of the University of Pavia for the kind hospitality during his visit.

\section{References}

[1] M. Bisi, J.A. Carrillo, G. Toscani. Contractive Metrics for a Boltzmann equation for granular gases: Diffusive equilibria. J. Stat. Phys. 118 (1-2) 301-331 (2005).

[2] M. Bisi, J.A. Carrillo, G. Toscani. Decay rates in probability metrics towards homogeneous cooling states for the inelastic Maxwell model. J. Stat. Phys. 124 (2-4) 625-653 (2006).

[3] A.V. Bobylev. The theory of the spatially Uniform Boltzmann equation for Maxwell molecules. Sov. Sci. Review C 7, 112-229 (1988). 
[4] A.V. Bobylev, J.A. Carrillo, I. Gamba. On some properties of kinetic and hydrodynamics equations for inelastic interactions. J. Stat. Phys. 98, 743-773 (2000).

[5] A.V. Bobylev, C. Cercignani. Self-similar asymptotics for the Boltzmann equation with inelastic and elastic interactions. J. Stat. Phys. 110, 333-375 (2003).

[6] A.V. Bobylev, I.M. Gamba and V. Panferov. Moment inequalities and high-energy tails for Boltzmann equations wiht inelastic interactions. J. Stat. Phys. 116 1651-1682 (2004).

[7] J.P. Bouchaud, M. Mézard. Wealth condensation in a simple model of economy. Physica A 282, 536-545 (2000).

[8] J.A. Carrillo, G. Toscani. Contractive probability metrics and asymptotic behavior of dissipative kinetic equations. Riv. Mat. Univ. Parma 6, 75-198 (2007).

[9] C. Cercignani, R. Illner, M. Pulvirenti. The mathematical theory of dilute gases. Springer Series in Applied Mathematical Sciences, Vol. 106 Springer-Verlag 1994.

[10] A. Chakraborti. Distributions of money in models of market economy. Int. J. Modern Phys. C 13, 1315-1321 (2002).

[11] A. Chakraborti, B.K. Chakrabarti. Statistical mechanics of money: how saving propensity affects its distributions. Eur. Phys. J. B. 17, 167-170 (2000).

[12] A. Chatterjee, B.K. Chakrabarti, S.S. Manna. Pareto law in a kinetic model of market with random saving propensity. Physica A 335, 155-163 (2004).

[13] A. Chakraborti, G. Germano, E. Heinsalu, M. Patriarca. Relaxation in statistical many-agent economy models. Eur. Phys. J. B. 57, 219-224 (2007).

[14] S. Cordier, L. Pareschi, G. Toscani. On a kinetic model for a simple market economy. J. Stat. Phys. 120, 253-277 (2005).

[15] A. Das, S. Yarlagadda. Analytic treatment of a trading market model. Physica Scripta T 106, 39-40 (2003).

[16] P. Degond, L. Pareschi, G. Russo. Modelling and numerical methods for kinetic equations. Birkhäuser, Boston, MA, 2004.

[17] L. Desvillettes, G. Furioli, E. Terraneo. Propagation of Gevrey regularity for solutions of Boltzmann equation for Maxwellian molecules. Trans. Amer. Math. Soc. (in press).

[18] T. Di Matteo, T. Aste, S.T. Hyde. in: The physics of complex systems (New advances and perspectives) F, Mallamace \& H.E. Stanley eds., IOS press, Amsterdam, 2004.

[19] N. Ding, N. Xi, Y. Wang. Effects of saving and spending patterns on holding time distribution. The European Physical Journal B 36, 149-153 (2003).

[20] A. Drăgulescu, V.M. Yakovenko. Statistical mechanics of money. Eur. Phys. Jour. B 17, 723729 (2000). 
[21] A. Drăgulescu, V.M. Yakovenko. Exponential and power law probability distribution of wealth and income in the United kingdom and the United states. Physica A 299, 213-221 (2001).

[22] B. Düring, G. Toscani. Hydrodynamics from kinetic models of conservative economies. Physica A 384, 493-506 (2007).

[23] M.H. Ernst, R. Brito. High energy tails for inelastic Maxwell models. Europhys. Lett 43, 497-502 (2002).

[24] M.H. Ernst, R. Brito. Scaling solutions of inelastic Boltzmann equation with over-populated high energy tails. J. Stat. Phys. 109, 407-432 (2002).

[25] X. Gabaix, P. Gopikrishnan, V. Plerou, H. E. Stanley. A Theory of Power-Law Distributions in Financial Market Fluctuations. Nature 423, 267-270 (2003).

[26] E. Gabetta, G. Toscani, B. Wennberg. Metrics for probability distributions and the trend to equilibrium for solutions of the Boltzmann equation. J. Statist. Phys. 81, 901-934 (1995).

[27] I.M. Gamba, S. Rjasanow, W. Wagner. Direct simulation of the uniformly heated granular Boltzmann equation. Math. Comput. Modelling 42, 683-700 (2005).

[28] L. Gosse, G. Toscani. Identification of asymptotic decay to self-similarity for onedimensional filtration equations. SIAM J. Numer. Anal. 43 (6), 2590-2606 (2006).

[29] B. Hayes. Follow the money. American Scientist 90 (5), 400-405 (2002).

[30] S. Ispolatov, P.L. Krapivsky, S. Redner. Wealth distributions in asset exchange models. Eur. Phys. Jour. B 2, 267-276 (1998).

[31] M. Kac. Probability and related topics in the physical sciences. Interscience Publishers, London-New York 1959.

[32] R.G. Laha, V.K. Rohatgi. Probability Theory. John Wiley and Sons, New York, 1979.

[33] M. Levy, S. Solomon. New evidence for the power-law distribution of wealth. Physica A 242 , 90-94 (1997).

[34] H. Levy, M. Levy, S. Solomon. Microscopic simulations of financial markets. Academic Press, New York, 2000.

[35] O. Malcai, O. Biham, S. Solomon, P. Richmond. Theoretical analysis and simulations of the generalized Lotka-Volterra model. Phys. Rev. E 66, 031102 (2002).

[36] B. Mandelbrot. The Pareto-Lévy law and the distribution of income. International Economic Review 1, 79-106 (1960).

[37] D. Matthes, G. Toscani. Analysis of a model for wealth redistribution. Preprint (2007). 
[38] P.K. Mohanty. Generic features of the wealth distribution in an ideal-gas-like market. Phys. Rev. E 74 (1) 011117 (2006).

[39] L. Pareschi, G. Toscani. Self-similarity and power-like tails in nonconservative kinetic models. J. Stat. Phys. 124 (2-4) 747-779 (2006).

[40] V. Pareto. Cours d'Economie Politique. Lausanne and Paris, 1897.

[41] M. Patriarca, A. Chakraborti, K. Kaski. Statistical model with a standard $\Gamma$ distribution. Physical Review E 70, 016104 (2004).

[42] V. Plerou, P. Gopikrishnan, H.E. Stanley. Econophysics: Two-Phase Behaviour of Financial Markets. Nature 421, 130 (2003).

[43] A. Pulvirenti, G. Toscani. Asymptotic properties of the inelastic Kac model. J. Stat. Phys. 114, 1453-1480 (2004).

[44] P. Repetowicz, S. Hutzler, P. Richmond. Dynamics of money and income distributions. Physica A 356 (2-4), 641-654 (2005).

[45] S. Rjasanow. Monte-Carlo methods for the Boltzmann equation. in: Modeling and computational methods for kinetic equations, 81-115. Model. Simul. Sci. Eng. Technol., Birkhäuser, Boston, MA, 2004.

[46] S. Sinha. The Rich Are Different!: Pareto Law from asymmetric interactions in asset exchange models, in: Econophysics of Wealth Distributions, 177-184 eds. A. Chatterjee, B. K. Chakrabarti and S. Yarlagadda, Springer Verlag, New York 2005.

[47] F. Slanina. Inelastically scattering particles and wealth distribution in an open economy. Phys. Rev. E 69, 046102 (2004).

[48] S. Solomon. Stochastic Lotka-Volterra systems of competing auto-catalytic agents lead generically to truncated Pareto power wealth distribution, truncated Levy distribution of market returns, clustered volatility, booms and crashes. Computational Finance 97, eds. A-P. N. Refenes, A.N. Burgess, J.E. Moody. Kluwer Academic Publishers, Dordrecht 1998. 